This is a post-peer-review, pre-copyedit version of an article published in Journal of Systems Science and Systems Engineering. The final authenticated version is available online at: https://link.springer.com/article/10.1007/s11518$\underline{015-5262-4}$

\title{
MULTIOBJECTIVE ROUTING IN MULTISERVICE MPLS NETWORKS WITH TRAFFIC SPLITTING - A NETWORK FLOW APPROACH
}

\author{
Rita Girão-Silva $^{1,2} \quad$ José Craveirinha $^{2} \quad$ João Clímaco $^{2} \quad$ M. Eugénia Captivo $^{3}$ \\ ${ }^{I}$ Department of Electrical and Computer Engineering, University of Coimbra \\ Pólo II, Pinhal de Marrocos; P-3030-290 Coimbra; Portugal \\ rita@deec.uc.pt \\ ${ }^{2}$ Institute of Computers and Systems Engineering of Coimbra (INESC-Coimbra) \\ R. Antero de Quental, 199; P-3000-033 Coimbra; Portugal \\ jcrav@deec.uc.pt_jclimaco@inescc.pt \\ ${ }^{3}$ Centro de Investigação Operacional, Faculdade de Ciências, Universidade de Lisboa
}

Campo Grande, Bloco C6, Piso 4; P-1749-016 Lisboa; Portugal

mecaptivo@fc.ul.pt

\begin{abstract}
A multiobjective routing model for Multiprotocol Label Switching networks with multiple service types and traffic splitting is presented in this paper. The routing problem is formulated as a multiobjective mixed-integer program, where the considered objectives are the minimization of the bandwidth routing cost and the minimization of the load cost in the network links with a constraint on the maximal splitting of traffic trunks. Two different exact methods are developed for solving the formulated problem, one based on the classical constraint method and another based on a modified constraint method. A very extensive experimental study, with results on network performance measures in various reference test networks and in randomly generated networks, is also presented and its results are discussed.
\end{abstract}

Keywords: Routing models, Multiobjective optimization, Telecommunication networks, Network flow approach, Traffic splitting.

\section{Introduction}

The routing calculation and optimization problems in modern multiservice networks are quite challenging, as the performance and cost metrics in these networks are multi-dimensional and often conflicting. Routing problems in communication networks consist in the selection of a sequence of network resources (i.e. paths or 'routes') that seek the optimization of some objective functions, while satisfying a set of constraints. According to the route related network metrics which are chosen, the performance of different routing decisions may be measured and compared.

There are potential advantages in formulating routing problems in multiservice networks as multiple 
objective optimization problems, because the trade-offs among distinct performance metrics and other network cost function(s), potentially conflicting, can be analyzed in a consistent manner.

In a Multiprotocol Label Switching (MPLS) network, packets are forwarded through LSPs (Label Switched Paths), according to certain technical rules. An important problem in traffic engineering is to distribute the traffic trunks (i.e. the aggregation of traffic flows between a pair of nodes/routers of the same FEC - Forwarding Equivalence Class) on the network by the possible LSPs, in an adequate manner. This procedure is known as traffic splitting, as the traffic trunks are split and mapped onto different paths in the network, satisfying the constraints of the bandwidth required by the traffic trunk of the given service class. This is useful to obtain a balanced distribution of the load in the network, but it entails the establishment of more LSPs and an increase in the complexity of the network management.

Some works concerned with load balancing can be mentioned. A multiobjective problem is formulated in [40] in the context of off-line routing in telecommunication networks. The multimedia traffic flows in current networks are characterized in [28] as batch Markovian arrival processes. The authors show that when these traffic flows are split, the whole network performance (measured in terms of end-to-end delay, delay variance, and cell loss probability) tends to improve. A survey on different multipath routing techniques in the Internet is presented in [38]. A fine grained traffic splitting technique is used in [23] in data center networks providing a better load-balanced network, when compared to networks where equal-cost multipath routing is considered.

An overview on load balancing techniques in IP/MPLS networks can be found in [65]. Also see [44] for a comparison on multipath routing algorithms for MPLS networks, where the traffic demand is distributed among selected candidate paths so that an adequate balancing of the load in the network is achieved. A survey on different multipath routing techniques, including traffic splitting, is proposed in [42].

Many traffic splitting algorithms proposed for MPLS networks concerned with an appropriate load distribution can be mentioned. A multipath adaptive traffic engineering scheme (MATE) is proposed in [26]. In this scheme, the load is adaptively balanced among multiple paths based on measurement and analysis of path congestion. A multipath routing approach which adapts itself in different time scales, to the changing network conditions, is presented in [57]. Global information for selecting a few good paths based on their long term available bandwidths is used, together with local information to cushion the short term variations in their available bandwidths. The paper [43] proposes a traffic engineering algorithm that calculates the traffic split ratio for multiple paths, under constraints such as maximum hop count, and preferred or not preferred node/link list. A dynamic multipath traffic engineering mechanism named LDM (Load Distribution over Multipath) is proposed in [66]. The traffic is distributed among multiple paths based on both the length and the load of a path, so as to enhance the network utilization as well as the network performance. A traffic splitting scheme is proposed in [45] for MPLS networks. The traffic is split among multiple candidate paths, previously selected having in mind information on the widest disjoint paths. The purpose of this scheme is to guarantee an adequate load distribution over the network. A traffic splitting mechanism to improve network security (in case of malicious attacks) 
and resource utilization in MPLS networks is proposed in [3]. A variable splitting ratio based algorithm is proposed in [55], where the load is balanced by an iterative method. In every iteration, a part of the load is split from the maximum cost path to the minimum cost path.

Other traffic splitting algorithms proposed in MPLS networks can be mentioned. The problem of allocating bandwidth to competing flows in an MPLS network, subject to constraints on fairness, efficiency, and administrative complexity is considered in [71]. In this model of traffic splitting, each flow is assigned a preferred or primary LSP, but traffic may be sent to other secondary LSPs, if needed. The preferred assignment is the one that sends most traffic of a source on the primary path of that source and minimizes the amount of traffic sent over secondary paths. In a context of fault recovery in MPLS networks, a model with traffic splitting is described in $[18,19]$. In a case of LSP or link failure, the associated traffic is split and rerouted to a set of alternative pre-assigned LSP paths, so that the total traffic throughput and the resource utilization are maximized. The amount of traffic to be forwarded on each pre-assigned backup LSP is calculated off-line considering a case based reasoning process where information on the past experience of the loading process is taken into account. In [41], a multipath load balancing algorithm is used to optimally split the incoming traffic in MPLS networks. A composite cost function representing the combined effect of average delay and jitter in the network is used, in order to express a measure of the Quality of Experience (QoE) perceived by the end user. Another multiobjective routing optimization model using two disjoint paths per flow is in [16], where a hierarchical multicriteria routing optimization formulation is proposed. The formulated problem is solved exactly by recurring to an adaptation of the algorithm for calculating non-dominated bi-criteria shortest pairs of disjoint paths given in [12].

We consider in this paper a multiobjective formulation for the global routing problem i.e. involving the simultaneous calculation of the LSPs for all node-to-node traffic flows, where traffic splitting is allowed. This type of routing approach may be considered as a specific type of network-wide optimization in the sense defined in [54, 17], since the objective functions of the route optimization model depend explicitly on all traffic flows in the network. Note that the traffic splitting models reviewed in the previous paragraphs normally calculate the traffic splitting and the associated paths on a per flow basis, that is, for each end-to-end offered flow at a time and unlike our model, they may be considered as flow-oriented optimization approaches. In contrast, network-wide optimization approaches such as the one addressed in this paper perform global optimization by executing the joint calculation of traffic splits for all traffic flows simultaneously, so as to obtain Pareto optimal solutions (see definition later in this section) to the chosen network performance/cost objective functions. A further discussion on this and related modeling and methodological issues can be seen in [17].

Some authors have tackled similar types of formulations. We can mention the works [54, 27]. A biobjective lexicographic routing problem is formulated in [54], where the objectives are the maximization of the QoS (Quality of Service) traffic revenue and of the Best Effort (BE) traffic revenue. Traffic splitting is allowed in this context. The problem is solved by a lexicographic optimization method, where only the QoS traffic is considered first, and the BE flows will take only the remaining available 
bandwidth. A model with three objectives (including the minimization of traffic splitting) is considered in [27]. The formulated routing problem is a bi-objective one, with a constraint on the total number of paths used in the network, and it is solved by a lexicographic weighted Chebyshev metric method.

A stochastic multiobjective routing formulation framework in the context of MPLS networks was proposed in [17]. This meta-model is a network-wide optimization routing approach, with multiple and hierarchical objectives and considering two different service classes, QoS and BE, and different types of services in each class. The possibility of alternative routing is also considered. A specialized heuristic strategy for finding adequate compromise solutions to a specific simplified variant of the optimization meta-model is proposed in [34].

A multiobjective model allows a mathematically consistent evaluation of the trade-offs between the considered objectives. In multiobjective optimization problems, see e.g. [69], one seeks to find nondominated solutions, i.e. feasible solutions such that it is not possible to improve the value of an objective function without worsening the value of at least one of the other objective functions. The non-dominated solutions may also be designated as Pareto optimal solutions and their set constitutes the so-called Pareto front. A review on multicriteria routing optimization models for modern communication networks with a case study can be seen in [11]. A proposal of a conceptual framework for consistent multicriteria routing approaches in QoS-IP networks is given in [74].

The routing problem considered here is formulated as a multiobjective mixed-integer program, where the considered objectives are the minimization of the bandwidth transport cost and the minimization of the load cost in the network links, with a constraint on the maximal splitting of traffic trunks. The basic problem addressed here is an extension to a multiservice situation of the problem proposed in [27]. Furthermore, we have solved variants of the main problem by exact methods, that is, methods that can find any (exactly) non-dominated solutions and allow for a detailed analysis of the Pareto front of the multiobjective problem.

Several classical exact methods are presented in [13], namely the weighting method, the constraint method, the non-inferior set estimation method and the multiobjective simplex method. An annotated bibliography of multiple objective combinatorial optimization problems is provided in [25], including a section on available exact methods. Some exact methods are also mentioned in [53], such as the physical programming method, the normal boundary intersection method and the compromise programming method. A variant of the constraint method, designated in [53] as a 'normalized normal constraint method', is also described. The work [51] focuses on the constraint method to solve multiobjective mathematical programming problems and presents a version of the method that avoids weakly nondominated solutions and seeks to accelerate the resolution process.

Concerning the methodological approaches that can be used for tackling multiobjective mixed integer programming problems, when exact methods are too costly in terms of execution time or memory requirements, heuristics and metaheuristics of various types may be considered. This is especially important in situations where the execution time is too high for the application purposes. Examples are dynamic routing models with short routing update periods or on-line routing models, where the routing 
solutions have to be calculated in times of the order of a few ms.

There is a vast number of bibliographical references regarding heuristics and metaheuristics for the resolution of multiobjective problems, for instance $[31,70]$. Some of the major types of metaheuristics include: (i) local search, where the space of candidate solutions is searched to try and find a solution which optimizes a specific criterion among a number of candidate solutions [1]; (ii) tabu search, where a local search is performed in the neighborhood of a found local optimum, while using memory structures that prevent going back to already visited solutions or allow for the establishment of user-provided rules [32]; (iii) simulated annealing, where the physical process of slowly cooling a certain material is replicated, in the sense that the solution space is extensively explored, and as the possible solutions are examined there is a decreasing probability of accepting worse solutions [5]. Although some of these metaheuristics may be adapted for use in multiobjective optimization, an important and vast line of investigation in this field focuses on Evolutionary Algorithms (EAs) [20, 33, 8, 77] and [76 - section I]. An extensive review on Multiobjective Evolutionary Algorithms (MOEAs) available in the literature is beyond the scope of this paper, but we will mention an approach of this type, which seems quite promising in the field of MOEAs: the Multiobjective Evolutionary Algorithm based on Decomposition (MOEA/D) algorithm proposed in [76], which is an EA, i.e. a population-based metaheuristic optimization algorithm that tries to find potentially non-dominated solutions. The main feature of this EA is the decomposition of a multiobjective optimization problem into several scalar optimization subproblems, which will be optimized simultaneously. Therefore, issues such as fitness assignment and diversity maintenance become easier to deal with. In the optimization of each subproblem, information from the neighboring subproblems is used, which leads to a lower computational complexity than other current MOEAs.

Note that the solutions obtained with heuristic and metaheuristic approaches are not guaranteed to be non-dominated solutions of the multiobjective problems. In fact, each obtained solution may be nondominated in relation to all the other solutions found throughout the execution of the algorithms and potentially, but not necessarily, non-dominated per se. The set of solutions may constitute an approximation to the Pareto front. In the case of our problem and our application study concerning the reference test networks, the developed exact resolution methods are computationally feasible and therefore their use for resolution purposes is fully justified, as they guarantee that all the obtained solutions are indeed non-dominated per se and are part of the Pareto front.

A final note concerning the fact that there are some types of problems in different application environments with some similarities to the problem addressed in this paper. Consider in particular the problem of routing and traffic assignment in transport networks. The total traffic between each origin-destination pair also has to be considered, along with the capacity of each road of the network. This problem has also been studied by many authors as a multi-objective problem, with many different resolution approaches. The most common approach to dealing with this problem is by considering two objectives: travel time and travel associated toll costs. These objectives are conflicting, as faster routes will have a higher toll cost associated with it. A value of time (VOT) is considered for each user, depending on how 
much money or time that user spends on a particular trip. Nevertheless, important differences (with respect to the type of problem addressed in this paper) concerning the representation of the traffic flows, the nature and formulation of the objective functions and some constraints can be identified, since these are specific to the application environment (communication networks using MPLS as the basic information transport technology in our case). Naturally, these features may have a significant impact in the specific mathematical formulations and resolution algorithms that are used in both cases.

An extensive overview of contributions in the area of routing problems in transport networks is out of the scope of our work, but some reviews on routing and traffic assignment optimization in transport networks can be seen in $[64,58,63]$, and more recently in $[60,50,37,6,60,46,9]$. We will now make reference, in particular, to works concerning multiobjective formulations of routing problems in transport networks. The objectives of cost and time are considered in many references. Work on this problem has been developed in $[21,22]$, where a probabilistic distribution is considered for the VOT parameter, rather than a fixed value. In $[75,39]$, a VOT is associated with the users of a specific class. As different classes of users experience different trade-offs between time and money (as they have different VOTs), an analysis on multiclass bi-criterion traffic equilibrium models is presented [75]. A multiclass, multicriteria logit-based traffic equilibrium assignment model in road networks served by advanced traveler information systems (ATIS) is presented in [39]. In [2], the route cost functions are nonadditive functions of time, where the cost parameter is converted to a time parameter. In [49], users' path choices are considered in response to time-varying toll charges. Therefore, heterogeneous users with different VOT preferences are taken into account in the underlying path choice decision framework. A simulation-based heuristic approach is proposed to find an adequate path flow pattern in the bicriteria dynamic user equilibrium problem. We can also cite [62], where the authors propose heuristic solution methods that allow to obtain equilibrium solutions to the traffic assignment problem. An approach to model the stochastic route choice in a tolled road network is proposed in $[72,73]$. All the users try to minimize both their travel time and the paid toll cost, in the context of a set of preferences represented by an indifference function: associated with a path with a specific toll, there is a limit on the time a user is willing to take. An additional third criterion (besides cost and time) is considered in [56], where a multiclass multicriteria network equilibrium problem is proposed. The criteria are travel time, travel cost and the emissions generated. Class-dependent and link-dependent weights are associated with each criterion.

Another relevant paper regarding the subject of path-based traffic assignment is [4]. In this paper, a set of paths is obtained prior to the traffic assignment, and heuristic rules are proposed in order to confine the path set to a reasonable size. The papers $[47,48]$ are also relevant, as they propose an alternate formulation of the traffic assignment problem, by defining a gap function to convert a Nonlinear Complementarity Problem (NCP) formulation of the user-equilibrium traffic assignment problem to an equivalent Mathematical Program (MP) formulation, which allows for the use of a large number of solution techniques developed for MP. Different solution approaches are proposed: one based on a set of working routes modeled according to the users' preferences; another based on a column generation 
procedure to generate a new route in each iteration. A framework is proposed in [7] for the design of an optimal multimodal transport network, where multiple objectives are considered. In this reference, a bilevel optimization is performed, with an upper level regarding the behavior of the planner or government and a lower level regarding the behavior of the traveler.

The main contributions of this paper are the following:

- formulation (in the context of a network-wide multiobjective optimization routing approach) of a multiobjective routing model for MPLS networks with traffic splitting and multiple service types, where the considered objectives are the minimization of the bandwidth routing cost and the minimization of the load cost in the network links with a constraint on the maximal number of paths that may be used by any flow;

- development of two different exact methods for solving the aforementioned model adapted to the application environment: the first one based on the classical constraint method [13] (identified hereafter as $M C C$ or Multi-Criteria Classical constraint method) and the second one based on a modified constraint method proposed in [53] (identified hereafter as $M C M$ or Multi-Criteria Modified constraint method). We have added to these methods special features related to the nature of the application, one that allows the choice of a specific area of the Pareto front to be explored and where more interesting solutions can be found, and another that allows for the choice of a specific solution as an adequate solution to the routing problem. Both features rely on a system of preferences established through the definition of preference regions in the objective function space obtained from aspiration and reservation levels (preference thresholds) defined for the considered objective functions;

- a very extensive experimental study, where many different reference networks are considered, with different traffic offered values (including randomly generated values) [27, 52, 54, 59, 67, 68]. Further experiments were also carried out with a set of randomly generated networks obtained with the gt-itm (Georgia Tech - Internetwork Topology Models) software [78]. In these cases, a double randomness is considered, as the MPLS networks topologies are randomly generated and the matrix of the traffic offered to those networks is also randomly generated;

- analysis of the results obtained for relevant network performance measures (based on the ones presented in [68]). Some of the performance measures are related to the links utilization, as this is a very important parameter concerning network traffic carrying capabilities. Other performance measures allow for a comparison of the final solutions with the ideal solutions that would be obtained if a single objective problem was solved. Results on these performance measures were obtained and a statistical study on their average values and variation ranges was performed.

The paper is organized as follows: in the next section, the routing model and the mathematical formulation of the multiobjective optimization problem are described. In section 3, two methods for solving the proposed problem are presented. A fourth section describing the considered experimental study and the obtained results is followed by a final section with some conclusions and an outline of future work. 


\section{Model Description}

The notation of the model and the multiobjective mathematical programming formulations of the multiobjective routing model for MPLS networks with traffic splitting, are described next.

\subsection{Nomenclature and Notation}

A network $(N, A)$ with unidirectional arcs (or links) is considered, where $N$ is the set of nodes in the network and $A$ is the set of links in the network. The capacity of each network link is given by $u_{k}[\mathrm{Mbit} / \mathrm{s}], k \in A$.

We consider different types of services and $S$ denotes the set of services of the network. Considering the point-to-point offered bandwidth matrix and the percentage of bandwidth associated with each service $\left(q_{s}, s \in S\right.$, with $\left.\sum_{s \in S} q_{s}=1.0\right)$ are given, the value of the bandwidth that is offered by each flow to the network may be calculated. Let $T_{i j}[\mathrm{Mbit} / \mathrm{s}]$ be the total value of bandwidth that is effectively offered from node $i$ to node $j$ in the network. The average bandwidth offered by the flow $t \equiv(i, j, s) \quad$ (corresponding to the traffic from service $s \in S$ originating in node $i$ and destined to node $j$ ) is $d_{t}=q_{s} T_{i j}$. The set of all network flows is $T$. For each flow $t$, a set of $L_{t}$ feasible paths is considered, $P_{t}=\left\{p_{t}^{0}, p_{t}^{1}, \ldots, p_{t}^{L_{t}-1}\right\}$. Traffic splitting is allowed in this model, i.e. the bandwidth that is offered by a flow $t$ to the network may be divided by $L_{t}$ feasible paths.

\subsection{Objective Functions}

For each network link $k \in A$, an additive cost per unit of bandwidth, $c_{k}$, is considered; $C_{t}^{l}$ is the cost of using path $p_{t}^{l}$, the $l$-th feasible path for flow $t$; the decision variable $x_{t}^{l}$ is the part of the bandwidth offered by flow $t$ that will be carried in the $l$-th path.

The first objective function is the minimization of the total cost of carrying the bandwidth of all the flows offered to all the feasible paths (similarly to the objective function A proposed in [68]):

$$
\min F_{1}=\sum_{t \in T} \sum_{l=0}^{L_{t}-1} C_{t}^{l} x_{t}^{l}
$$

with

$$
\begin{aligned}
& C_{t}^{l}=\sum_{k \in p_{t}^{l}} c_{k}, \forall l=0, \ldots, L_{t}-1 ; t \in T \\
& \sum_{l=0}^{L_{t}-1} x_{t}^{l}=d_{t}, \forall t \in T \\
& x_{t}^{l} \geq 0, \forall l=0, \ldots, L_{t}-1 ; t \in T
\end{aligned}
$$

where the constraint (3) guarantees that the total bandwidth required by flow $t$ is carried by the assigned LSPs.

The second objective function in this problem is the minimization of the load cost in all the network links, due to the carrying of the bandwidth of all the flows offered to all the feasible paths that are used. The aim is to avoid the over-utilization of some of the links while others remain under-utilized. A more balanced distribution of load in the network may be accomplished, so as to maximize the possibility of the network accepting more traffic in the future. This type of function has been often used in earlier 
studies on routing models in MPLS (see examples in $[27]$ and $[15,16])$ and was originally proposed in [29, 30].

A piece-wise linear cost function $\phi_{k}, k \in A$ is based on the utilization rate of link $k$, i.e. the ratio between the total load $f_{k}$ carried in the link and the link capacity $u_{k}$. By using this function, sending packets over a link is increasingly penalized as the utilization of the link increases. The used calibration of this piece-wise linear function, for MPLS networks, results from earlier studies on routing in MPLS [27], aiming at maximizing the traffic carrying capacity of the network, in the event of future traffic increase. Hence, the second objective function is:

$$
\min F_{2}=\sum_{k \in A} \phi_{k}
$$

with

$$
\begin{aligned}
& \phi_{k} \geq f_{k}, \forall k \in A \\
& \phi_{k} \geq 2 f_{k}-0.5 u_{k}, \forall k \in A \\
& \phi_{k} \geq 5 f_{k}-2.3 u_{k}, \forall k \in A \\
& \phi_{k} \geq 15 f_{k}-9.3 u_{k}, \forall k \in A \\
& \phi_{k} \geq 60 f_{k}-45.3 u_{k}, \forall k \in A \\
& \phi_{k} \geq 300 f_{k}-261.3 u_{k}, \forall k \in A \\
& f_{k} \leq u_{k}, \forall k \in A \\
& f_{k}=\sum_{t \in T} \sum_{l=0}^{L_{t}-1} a_{t, l}^{k} x_{t}^{l}, \forall k \in A
\end{aligned}
$$

where (6)-(11) define the piece-wise linear function to be considered and (12) is the constraint that guarantees that the link capacity is not exceeded. The parameter $a_{t, l}^{k}$ is binary and defines whether a link $k$ belongs to path $p_{t}^{l}$, i.e. $a_{t, l}^{k}=1$ iff $k \in p_{t}^{l}, k \in A, l=0, \ldots, L_{t}-1, t \in T$ and $a_{t, l}^{k}=0$ otherwise.

The idea behind considering the cost function $\phi_{k}$ is that it is less expensive to send traffic over a link with a small utilization, as pointed out in [29]. As the utilization of a link increases, sending traffic over that link becomes more expensive, because of the sensitivity to sudden increases of traffic, hence limiting the traffic capabilities of the network. Therefore, the minimization of $F_{2}=\sum_{k \in A} \phi_{k}$ has to do with the objective of obtaining a globally balanced traffic distribution throughout the network, hence minimizing the probability of congestion.

The specific definition of $\phi_{k}$ is not important, as long as it is a piece-wise linear increasing and convex function, as claimed in [29]. Therefore, the specific piece-wise linear relationships may be defined for a specific routing problem, according to the network features and to the traffic demands, and cannot be readily generalized for any routing problem. We have considered the piece-wise linear relationships (6)-(11), as proposed in [27], for two main reasons: i) the links are not allowed to be used beyond their capacities; ii) the break points of the functions are at the utilization rates $f_{k} / u_{k}$ of the links given by $0.5,0.6,0.7,0.8$ and 0.9 , which are adequate for a telecommunications routing problem of this nature. 


\subsection{Constraints}

It is important to limit the number of used paths for each flow for technical reasons to prevent excessive overheads related to control and signaling costs. If the number of used paths per flow increases, then the network routing control and management may become increasingly costly and complex because the signaling and processing tasks increase.

Let $N_{L} \in \mathbb{N}$ be the maximal value allowed for the total number of paths used by any traffic flow. Let the binary variable $y_{t}^{l}$ define whether the path $p_{t}^{l}$ is used, i.e. $y_{t}^{l}=1$ iff the $l$-th path $p_{t}^{l}, l=0, \ldots, L_{t}-1$ is used by flow $t \in T$ and $y_{t}^{l}=0$ otherwise. Therefore, a constraint for this problem is

$$
\sum_{l=0}^{L_{t}-1} y_{t}^{l} \leq N_{L}, \forall t \in T
$$

with

$$
\begin{aligned}
x_{t}^{l} & \leq d_{t} y_{t}^{l}, \forall l=0, \ldots, L_{t}-1 ; t \in T \\
y_{t}^{l} & \in\{0,1\}, \forall l=0, \ldots, L_{t}-1 ; t \in T
\end{aligned}
$$

A constraint on the maximal number of links $D_{s}$ for the paths associated with a service $s \in S$ is also considered, in the terms that will be explained later.

\subsection{Problem Formulation}

The multiobjective routing problem $P_{0}$ to be addressed is

$\min \left\{F_{1}, F_{2}\right\}$

subject to: (3)-(4), (6)-(13), (14)-(16)

constraint on $D_{s}, \forall s \in S$

with $C_{t}^{l}$ calculated as in (2).

The total number $L_{t}$ of feasible paths for each flow $t$ is $L_{t}=\min \left\{N_{L}, N_{t}\right\}$. The maximal number of paths in the network for flow $t, N_{t}$, satisfies a constraint on the maximal number of links $D_{s}$ for the paths associated with service $s \in S$, which is usually defined for technical reasons, associated with transmission or traffic engineering and signaling requirements related to service type.

For the values of $D_{s}$, it is stipulated that: for flows of QoS services with real-time requirements (e.g. voice and video services), $D_{s}$ should be the network diameter (maximal number of links of the shortest paths for all the network pairs of nodes); for flows of QoS services without real-time requirements (e.g. Premium data services), $D_{s}$ should be the network diameter +1 ; for BE service flows (e.g. plain data services), no bounds on the maximal number of links are imposed, so $D_{s}=|N|-1$.

For the generation of the set $P_{t}$ for each flow $t$, the $K$-shortest path MPS algorithm in [36] was used.

For the calculation of the cost per unit of bandwidth in each link, an expression depending on the capacity of the link $u_{k}[\mathrm{Mbit} / \mathrm{s}]$ and its length $l_{k}[\mathrm{~km}]$ is considered: $c_{k}=\frac{\alpha}{u_{k}}+\beta l_{k}$, with $\alpha, \beta>0$. The first term reflects the economy of scale and the decrease in transmission times associated with 
increased capacity. The second term is related to propagation delays, which increase with the physical length of the link and are associated with specific transmission impairments.

The use of these two terms is quite common in the literature. We can cite [29], where it is mentioned that when the Open Shortest Path First (OSPF) routing protocol is used, the weights of the links can be set proportional to their physical distances and there are recommendations from network operators to consider the link weights inversely proportional to its capacity. Therefore, we tried to combine both aspects and devised a link cost function such as $c_{k}$.

The inclusion of the second term allows for the inclusion of a delay-related parameter, as we have explained. In fact, the transmission times tend to increase as the length of the links increases and network operators are of course interested in minimizing the end-to-end delay experienced by the traffic flows in the links.

Also note that by using this distance-based cost term, and multiplying it by a bandwidth parameter $\left(x_{t}^{l}\right)$ in function $F_{1}$, we get a bandwidth-distance product, which is used quite commonly in fiber transmission systems (as the effect of dispersion increases with the length of the fiber, there is a tradeoff between the bandwidth of the signal and the distance it can be carried). We can cite [52], where the bandwidth unit cost of a link is given precisely by a linear function of the length of the link.

As the two parameters in $c_{k}\left(u_{k}\right.$ and $\left.l_{k}\right)$ may have very different ranges of variation, we have used normalized versions of the parameters, $\frac{1}{u_{k}^{\prime}}$ and $l_{k}^{\prime}$, with $\frac{1}{u_{k}^{\prime}}=\frac{1 / u_{k}-\min _{\kappa \in A}\left(1 / u_{\kappa}\right)}{\max _{\kappa \in A}\left(1 / u_{\kappa}\right)-\min _{\kappa \in A}\left(1 / u_{\kappa}\right)}$ and $l_{k}^{\prime}=\frac{l_{k}-\min _{\kappa \in A}\left(l_{K}\right)}{\max _{\kappa \in A}\left(l_{\kappa}\right)-\min _{\kappa \in A}\left(l_{\kappa}\right)}$.

Hence, the actual expression for the cost per unit of bandwidth in each link is

$$
c^{\prime}{ }_{k}=\frac{\alpha}{u_{k}^{\prime}}+\beta l_{k}^{\prime}
$$

In the expression for $\frac{1}{u_{k}^{\prime}}$, it is assumed that not all the links in the network have the same capacity, i.e. $\max _{\kappa \in A} u_{\kappa} \neq \min _{\kappa \in A} u_{\kappa}$. If indeed all the links have the same capacity, then this parameter does no longer need to be included in the link cost, as it affects all the links in the same way. In this situation, we assume that $c^{\prime}{ }_{k}=\alpha+\beta l_{k}^{\prime}$.

\section{Resolution Methods}

The problem $P_{0}$ was solved by two algorithms described in this section, the first one based on the constraint method [13] and the other based on the modified constraint method [53]. For both methods, a feature for the exploration of a specific part of the Pareto front was added, allowing for the choice of an adequate non-dominated solution to the problem.

\subsection{Method Based on the Classical Constraint Method - MCC}

The first method used to solve problem $P_{0}$ is a method based on the classical constraint method [13]. In this method, only one objective will be optimized, while all the other objectives are constrained to some value. The optimal solution to this single objective problem is a non-dominated solution to the 
original multiobjective problem, provided some conditions are satisfied [13]. The bounds that are imposed on the constrained objectives have to be carefully chosen, so that a single optimal solution to the obtained single objective problem exists and so as to guarantee that different non-dominated solutions throughout the Pareto front may be obtained.

In figure 1, an example of the application of the $M C C$ method is presented. We consider a single objective problem of minimization of the objective function $F_{2}$, whereas a constraint is formulated for the other objective function, i.e. $F_{1} \leq F_{1 \mathrm{lim}}$. This constraint establishes a new feasible region where we seek to optimize $F_{2}$.

Notice that in this figure the extreme solutions of the Pareto front are shown, where $X$ is the solution $\left(F_{1}, F_{2}\right) \equiv\left(F_{1}^{\min }, F_{2}^{\max }\right)$ and $Y$ is the solution $\left(F_{1}, F_{2}\right) \equiv\left(F_{1}^{\max }, F_{2}^{\min }\right)$.

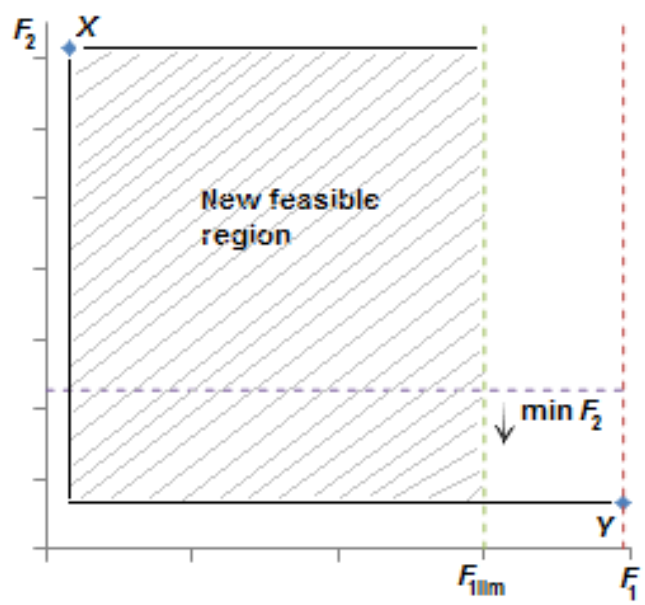

Figure 1 Example of the application of the MCC

The proposed method is described in the following algorithm:

\section{Algorithm $M C C$}

1. Definition of upper bounds $U B_{1}$ and $U B_{2}$ for both functions $F_{1}$ and $F_{2}$, respectively. Initially, both $U B_{1}$ and $U B_{2}$ are $\infty$.

2. Construction of a pay-off table for this problem. The problems of individual optimization of each of the objective functions $F_{1}$ and $F_{2}$ are solved at this step. Solving the problem of the optimization of $F_{1}$ will give the value of $F_{1}^{\min }$ and presumably $F_{2}^{\max }$, whereas solving the problem of the optimization of $F_{2}$ will give the value of $F_{2}^{\min }$ and presumably $F_{1}^{\max }$ as the functions $F_{1}$ and $F_{2}$ are usually conflicting.

There might be alternative optimal values for the problems of individual optimization of each of the objective functions in $P_{0}$. In that case, the obtained solutions might be weakly non-dominated in the objective function space. In order to guarantee that only non-dominated solutions are included in the 
pay-off table, the strategy proposed in [51] is used.

2.a) Solution of the auxiliary problem $P_{1}^{U}$ :

$\min \left\{F_{1}\right\}$

subject to: (3)-(4), (6)-(13), (14)-(16)

$$
\begin{aligned}
& F_{1} \leq U B_{1} \\
& F_{2} \leq U B_{2} \\
& \text { constraint on } D_{s}, \forall s \in S
\end{aligned}
$$

Let $F_{1}^{\text {min }}$ be the value of $F_{1}$ for the solution to this problem.

2.b) Solution of the auxiliary problem $P_{2}^{\prime U}$ :

$\min \left\{F_{2}\right\}$

subject to: $F_{1} \leq F_{1}^{\min }$

$$
\begin{aligned}
& (3)-(4),(6)-(13),(14)-(16) \\
& F_{1} \leq U B_{1} \\
& F_{2} \leq U B_{2} \\
& \text { constraint on } D_{s}, \forall s \in S
\end{aligned}
$$

Let $F_{2}^{\max }$ be the value of $F_{2}$ for the solution to this problem.

2.c) Solution of the auxiliary problem $P_{2}^{U}$ :

$$
\min \left\{F_{2}\right\}
$$

subject to: (3)-(4), (6)-(13), (14)-(16)

$$
\begin{aligned}
& F_{1} \leq U B_{1} \\
& F_{2} \leq U B_{2}
\end{aligned}
$$

$$
\text { constraint on } D_{s}, \forall s \in S
$$

Let $F_{2}^{\min }$ be the value of $F_{2}$ for the solution to this problem.

2.d) Solution of the auxiliary problem $P_{1}^{\prime U}$ :

$\min \left\{F_{1}\right\}$

subject to: $F_{2} \leq F_{2}^{\min }$

$$
\begin{aligned}
& (3)-(4),(6)-(13),(14)-(16) \\
& F_{1} \leq U B_{1} \\
& F_{2} \leq U B_{2} \\
& \text { constraint on } D_{s}, \forall s \in S
\end{aligned}
$$

Let $F_{1}^{\max }$ be the value of $F_{1}$ for the solution to this problem.

3. Solution of an auxiliary problem of mixed-integer type

3.a) Calculation of the range for $F_{1}: r_{1}=F_{1}^{\max }-F_{1}^{\min }$.

3.b) Definition of $\Delta$, the number of auxiliary constraints that will be considered.

3.c) For $\delta=1$ to $\delta=\Delta-2$ :

3.c)i. Calculate $N_{C}=F_{1}^{\max }-\frac{\delta}{\Delta-1} r_{1}$.

3.c)ii. Solution of the problem $P_{3}^{\prime}$ : 


$$
\min \left\{F_{2}-\gamma g\right\}
$$

subject to: $F_{1}+g=N_{C}$

$$
\begin{aligned}
& (3)-(4),(6)-(13),(14)-(16) \\
& F_{1} \leq U B_{1} \\
& F_{2} \leq U B_{2} \\
& g \geq 0 \\
& \text { constraint on } D_{s}, \forall s \in S
\end{aligned}
$$

with $\gamma \in \mathbb{R}^{+}$infinitely small.

Notice that the application of the $M C C$ would consist of solving a problem $P_{3}^{U}$, which would be similar to $P_{3}^{\prime U}$ except that we would try to minimize simply $F_{2}$ (rather than $F_{2}-\gamma g$ ) and the first constraint would be $F_{1} \leq N_{C}$ rather than $F_{1}+g=N_{C}$. An optimal solution to this problem $P_{3}^{U}$ corresponds to a non-dominated solution iff it is a unique optimal solution. Otherwise, the optimal solution obtained when solving $P_{3}^{U}$ could be one of various alternative optima and might correspond to a weakly non-dominated solution to problem $P_{0}$. In order to avoid this situation, problem $P_{3}^{\prime U}$ was formulated based on the method in [51] and solved.

3.d) The $\Delta$ solutions obtained when solving this problem $P_{3}^{\prime U}$ are non-dominated and so they are part of the Pareto front. In figure 2, an example of the result after steps 1 to 3 of the $M C C$ method is presented. A total of $\Delta$ solutions were found.

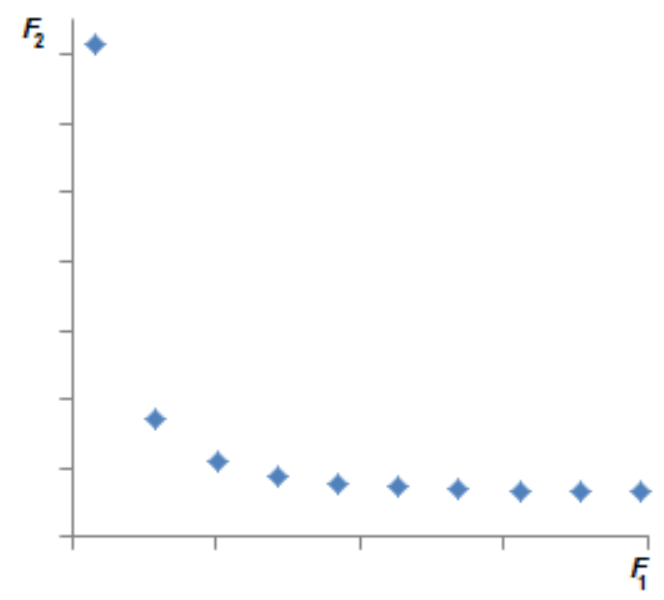

Figure 2 Example of the application of steps 1-3 of MCC, obtaining a total of $\Delta=10$ solutions that are part of the Pareto front

4. If the algorithm is being run for the first time, proceed to step 5. If the algorithm is being run for the second time, then a specific region of the Pareto front has already been thoroughly analyzed and we may 
proceed to step 7.

5. Choice of an area of the Pareto front that deserves to be more thoroughly analyzed, by considering preference regions in the bidimensional objective function space obtained from aspiration and reservation levels (preference thresholds) defined for the two objective functions: $F_{\zeta}^{r e q}=\frac{F_{\zeta}^{\min }+F_{\zeta}^{a v}}{2}$ and $F_{\zeta}^{a c}=\frac{F_{\zeta}^{\max }+F_{\zeta}^{a v}}{2}$ with $F_{\zeta}^{a v}=\frac{F_{\zeta}^{\min }+F_{\zeta}^{\max }}{2}, \quad \zeta=1,2$.

As an example of the definition of priority regions in the objective function space, see figure 3 . The ideal optimum is obtained when both objective functions $F_{1}$ and $F_{2}$ are optimized separately. The region for which the requested levels are satisfied for both objective functions is the first priority region $A$; the regions for which only one of the requested values is satisfied and an acceptable value is guaranteed for the other metric are the second priority regions $B_{1}$ and $B_{2}$ (note that when a choice has to be made between $B_{1}$ and $B_{2}$, we will consider $B_{1}$ slightly preferable to $B_{2}$ because, for solutions in any second priority region, a slight preference is given to the one with lower $F_{1}$ even if with greater $F_{2}$ ); the region where only acceptable values are guaranteed for both metrics is the third priority region $C$. Beyond the acceptable values, lies the least priority region $D$.

Therefore the new upper bounds $U B_{1}$ and $U B_{2}$ that will define an area of the Pareto front that will be looked into with more detail are defined by the boundaries of the region to be considered. Firstly, region $A$ will be considered, so $U B_{1}=F_{1}^{r e q}$ and $U B_{2}=F_{2}^{r e q}$; if there is no possible solution in region $A$, then region $B_{1}$ will be considered and $U B_{1}=F_{1}^{r e q}$ and $U B_{2}=F_{2}^{a c}$; and so on, exploring in succession, regions $B_{2}$ and $C$, if necessary.

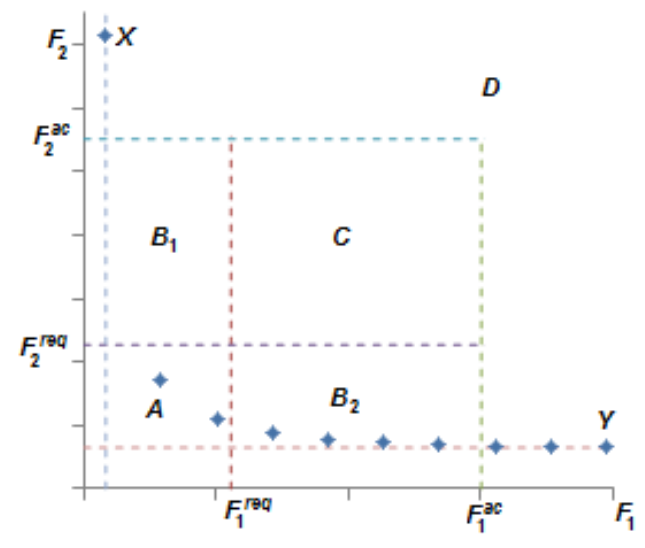

Figure 3 Example of the definition of priority regions in the objective function space (step 5 of $M C C$ )

6. Proceed to step 2.

7. Choice of an adequate non-dominated solution in the Pareto front, for which a Chebyshev weighted metric will be applied to the found non-dominated solutions. The approach chosen to select the "best" 
solution in the Pareto front relies on the minimization of a weighted Chebyshev distance to a reference point. As mentioned in step 5 of the algorithm, aspiration and reservation levels were specified for each criterion. In this context, the weighted Chebyshev distance of a non-dominated solution in a given preference region to an associated reference point (see definition below) is calculated and the "best" solution will be the one in the best possible priority region which minimizes that distance, following a method of the type described in [10]. With this approach, we are considering that in the best possible priority region (region $A$ ) both objective functions $F_{1}$ and $F_{2}$ have equal importance.

Defining $R$ as the best possible priority region in the objective function space where at least one solution $\rho$ can be found, a specific reference point $\left(C_{1 \mid R}^{*} ; C_{2 \mid R}^{*}\right)$ can be chosen in $R$ as the "ideal" point in that region. The reference point in each rectangular region is the bottom left corner of that region. For a non-rectangular region such as $D$, the considered reference point is the ideal optimum in the objective function space, $\left(F_{1}^{\min } ; F_{2}^{\min }\right)$.

Other parameters that must be defined are the minimum $m_{\zeta \mid R}$ and the maximal $M_{\zeta \mid R}$ values of each metric for region $R$.

The problem of selection of a final solution considers a weighted Chebyshev norm:

$$
\min _{\rho \in R} \max _{\zeta=1,2}\left\{w_{\zeta \mid R}\left|C_{\zeta}(\rho)-C_{\zeta \mid R}^{*}\right|\right\}
$$

where $C_{1}(\rho)=F_{1}(\rho)$ and $C_{2}(\rho)=F_{2}(\rho)$ are the metrics for solution $\rho$. The weights in the weighted Chebyshev distance, $w_{\zeta \mid R}=\frac{1}{M_{\zeta \mid R}-m_{\zeta \mid R}}$, allow the Chebyshev metrics $\left\{w_{\zeta \mid R}\left|C_{\zeta}(\rho)-C_{\zeta \mid R}^{*}\right|\right\}$ to be dimension free and proportional to the size of the rectangular region. This weighted Chebyshev norm seems to be the more adequate to the adopted technique of search and selection of non-dominated solutions in rectangular preference regions. In fact, the use of the weights (as defined in the method) makes the contour of the rectangle an isocost Chebyshev line for each particular region.

\subsection{Method Based on the Modified Constraint Method - MCM}

The second method used to solve problem $P_{0}$ is based on the modified constraint method developed in [53]. This method also relies on the formulation of a single objective problem related to the original multiobjective problem. As in the $M C C$, only one objective will be optimized, while constraints involving the other objectives are formulated.

Problem $P_{0}$ is bi-objective so we will optimize one of the objectives and formulate a constraint involving the other objective. We start by calculating the optimal solutions that would be obtained if each of the two objectives was taken separately, i.e. we calculate $F_{1}^{\min }$ and $F_{2}^{\min }$ in the feasible region. The line joining the two extreme points $X$ and $Y$ is called, in [53], the utopia line. The utopia line is divided in $\Delta-1$ segments, resulting in $\Delta$ points. In each of these points, a normal to the utopia line is considered, which will allow a reduction of the feasible space. Note that the points considered in the utopia line are evenly distributed. For each of the points defined in the utopia line, a constraint is formulated and an auxiliary single objective problem is solved. According to [53], when this method is used, there may occur situations where the optimal solution to this auxiliary problem does not correspond 
to a non-dominated solution in the original multiobjective problem and in these situations a so-called Pareto filter would be applied to the $\Delta$ solutions, so as to retain only the solutions which are not dominated by any other solution in the set of obtained solutions. However, in our problem these situations do not occur because the hull of the feasible solution set of our optimization problem is convex (as a result of the linearity properties of the objective functions) and therefore this Pareto filter was not applied.

The application of this method generates a set of solutions, which will tend to be a set of evenly distributed non-dominated solutions of the original multiobjective problem and constitute a good representation of the whole Pareto front (see as an example, figure 5).

In figure 4, an example of the application of the $M C M$ method is presented. Taking one of the points in the utopia line, the normal to the utopia line in that point is considered and it will allow us to formulate a constraint that restricts the feasible area. We then consider a single objective problem of minimization of the objective function $F_{2}$ in the new feasible area. Notice that in this figure the Pareto front is shown to clarify the example of the optimization of $F_{2}$ subject to the constraint that defines the new feasible area.

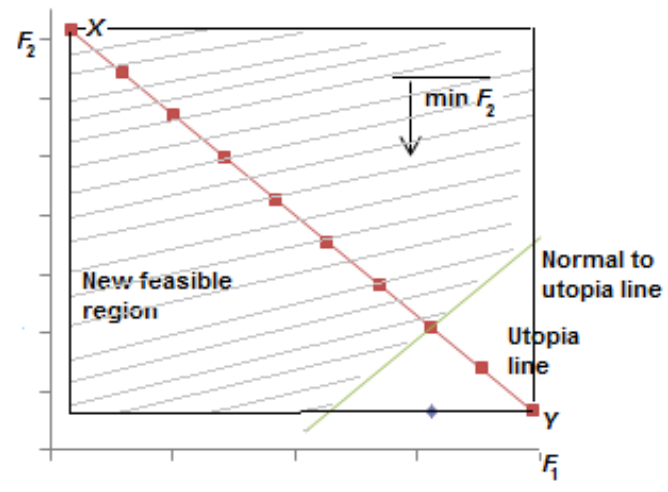

Figure 4 Example of the application of the MCM

A comparison between this figure and figure 1 shows clearly the difference between the two methods that we considered in our work. In the $M C C$ method, it is a normal to the $\mathrm{X}$ axis (rather than a normal to the utopia line) that will allow us to formulate the constraint that restricts the feasible area.

Notice that the utopia line is "closer" to the shape of the Pareto front than the X axis, and that is the reason why the consideration of the normal to the utopia line in the restriction of the feasible area will lead to finding solutions better distributed along the Pareto front. This can clearly be seen in figure 5: the comparison between this figure and figure 2 shows that in the $M C M$ method the solutions are indeed better distributed along the Pareto front.

Following the algorithm presented in the previous sub-section, only the changes to be performed in 
that algorithm will be mentioned here.

\section{Algorithm MCM}

1. (Step 1 as in the $M C C$ algorithm)

2. (Step 2 as in the $M C C$ algorithm)

3. Solution of an auxiliary problem of mixed-integer type

3.a) Calculation of the ranges for $F_{\zeta}$, with $\zeta=1,2: r_{\zeta}=F_{\zeta}^{\max }-F_{\zeta}^{\min }$.

3.b) Definition of $\Delta$, the number of auxiliary constraints that will be considered.

3.c) For $\delta=1$ to $\delta=\Delta-2$ :

3.c)i. Calculate $N_{\delta}=\frac{F_{1}^{\min }}{r_{1}}-\frac{F_{2}^{\min }}{r_{2}}+1-2 \frac{\delta}{\Delta-1}$.

3.c)ii. Solution of the problem $P_{4}^{\prime U}$ :

$\min \left\{F_{2}-\gamma g\right\}$

subject to: $\frac{F_{1}}{r_{1}}-\frac{F_{2}}{r_{2}}+g \leq N_{\delta}$

(3)-(4), (6)-(13), (14)-(16)

$$
\begin{aligned}
& F_{1} \leq U B_{1} \\
& F_{2} \leq U B_{2} \\
& g \geq 0 \\
& \text { constraint on } D_{s}, \forall s \in S
\end{aligned}
$$

with $\gamma \in \mathbb{R}^{+}$infinitely small.

Notice that the direct application of the $M C M$ would consist of solving a problem where we would try to minimize simply $F_{2}$ (rather than $F_{2}-\gamma g$ ). This perturbation of the objective function allows us to avoid potential weakly non-dominated solutions to problem $P_{0}$.

3.d) The solutions obtained are part of the Pareto front. In figure 5, an example of the result after steps 1 to 3 of the $M C M$ method is presented. A total of $\triangle$ solutions were found.

4. (Step 4 as in the $M C C$ algorithm)

5. (Step 5 as in the $M C C$ algorithm)

As an example of the definition of priority regions in the bidimensional objective function space of the obtained solutions in step 5 , see figure 6 .

6. (Step 6 as in the $M C C$ algorithm)

7. (Step 7 as in the $M C C$ algorithm)

Note that the proposed algorithms $M C C$ and $M C M$ enable that unsupported non-dominated solutions (i.e. non-dominated solutions located in the interior of the convex hull of the feasible solution set) may be found. 


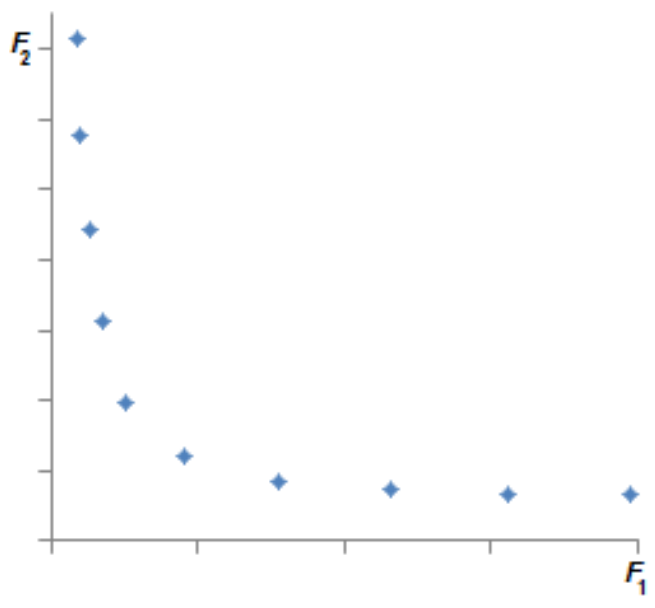

Figure 5 Example of the application of steps $1-3$ of $M C M$, obtaining a total of $\Delta=10$ solutions that are part of the Pareto front

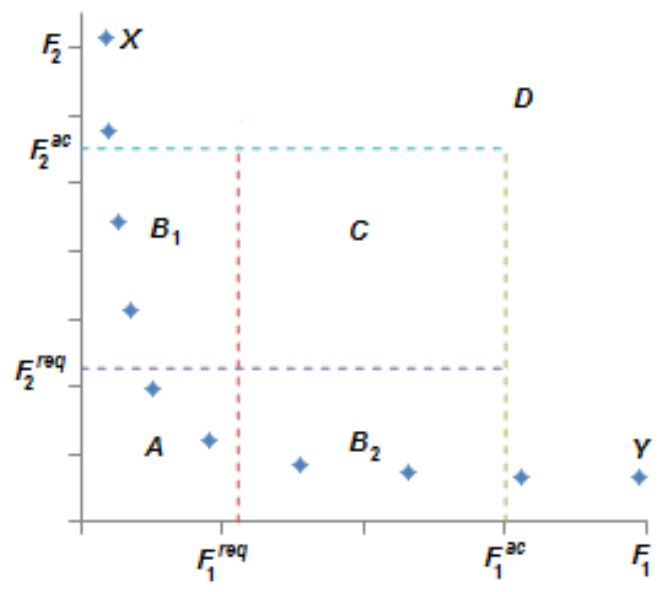

Figure 6 Example of the definition of priority regions in the objective function space (step 5 of $M C M$ )

\section{Experimental Study}

A very extensive experimental study was carried out. In the next sub-sections, the features of the networks and of the services are described. Afterwards, the performed tests are described and the network performance measures are presented. Finally, the obtained results are analyzed.

\subsection{Network Description}

Three different sets of networks N1, N2 and N3 were considered, as described next. 
The first set of networks, designated by N1, is a set of 5 networks presented in previous works [27, $54,59]$, where information on the capacities of the links and on the offered traffic is provided.

The first of these networks will be designated as N1-1a and is displayed in figure 7. It is considered in many different studies, e.g. [59, 67, 68]. It is a network with 12 nodes and 36 unidirectional links. Notice that in the original network, there are 18 bidirectional links, but we have applied a simple rule to transform them into unidirectional links, as we will explain later in the text. The average node degree is 3.0. The information on the capacities of the links and on the node-to-node offered traffic is provided by [59- N12-1].

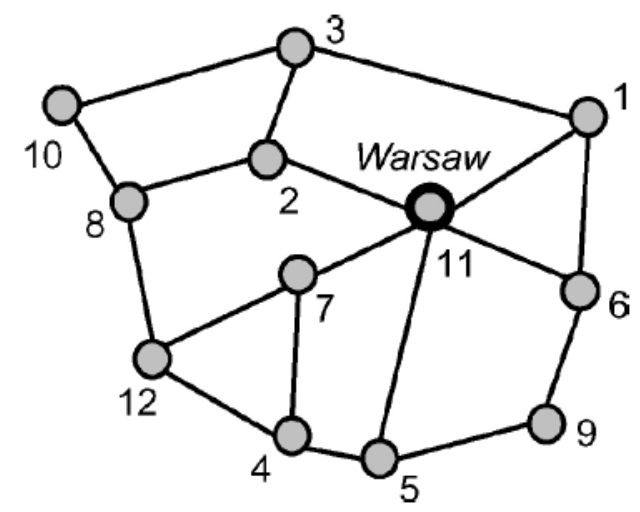

Figure 7 Network N1-1(a and b) and N2-1 [59- Fig. 7]

The second of these networks will be designated as N1-1b and has the same topology and the same demand volumes as N1-1a. The difference is on the capacities of the links, which are provided in [59N12-2].

The third of these networks will be designated as N1-2 and is displayed in figure 8. It is a network with 7 nodes and 24 unidirectional links. Notice that in the original network, there are 12 bidirectional links. The average node degree is 3.43. The information on the capacities of the links and on the nodeto-node offered traffic is provided in [59- N7].

The fourth of these networks will be designated as N1-3 and is displayed in figure 9. It is a network with 8 nodes and 20 unidirectional links, with an average node degree of 2.5 . The information on the capacities of the links and on the node-to-node offered traffic is provided in [54].

The last of these networks will be designated as N1-4 and is displayed in figure 10. It is a network with 10 nodes and 32 unidirectional links, with an average node degree of 3.2. The information on the capacities of the links and on the node-to-node offered traffic is provided in [27].

The second set of networks, designated by N2, is a set of 4 networks presented in [68]. Some of these networks have also been used in other studies, such as $[52,59,67]$. No information on the capacities of 


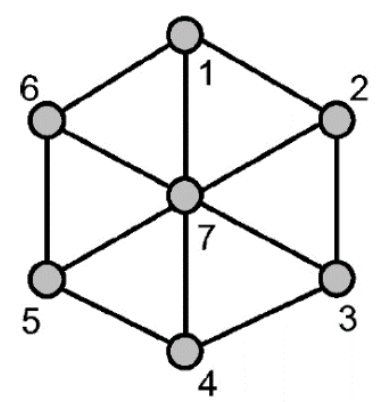

Figure 8 Network N1-2 [59 - Fig. 7]

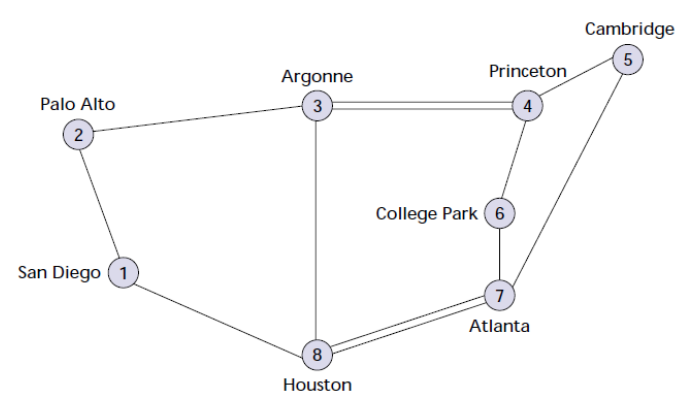

Figure 9 Network N1-3 [54 - Fig. 1]

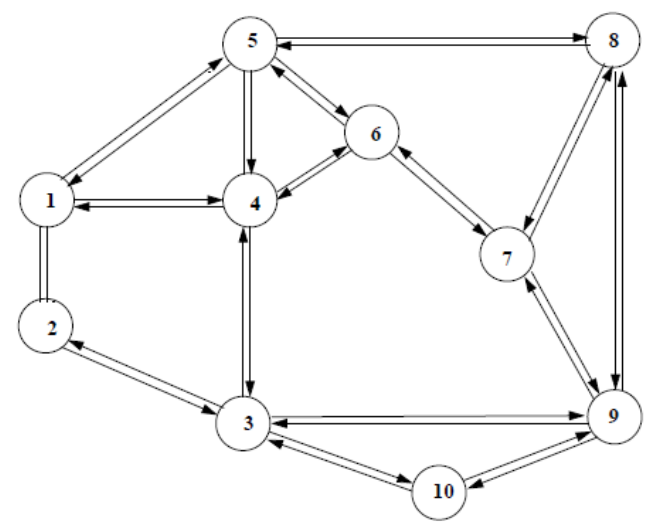

Figure 10 Network N1-4 [27]

the links or on the offered traffic is provided. We have followed the approach in [68] to estimate these parameters.

In these networks all the links are bidirectional but we have considered them unidirectional with the adaptation which will be explained later in the text.

The first of these networks will be designated as N2-1 and has the same topology as N1-1a and N1- 
$1 \mathrm{~b}$ (see figure 7). This network has also been used in [59, 67].

The second of these networks will be designated as N2-2 and is displayed in figure 11. It is a network with 6 nodes and 24 unidirectional links (12 bidirectional links in the original network), with an average node degree of 4.0. This network has also been used in [67].

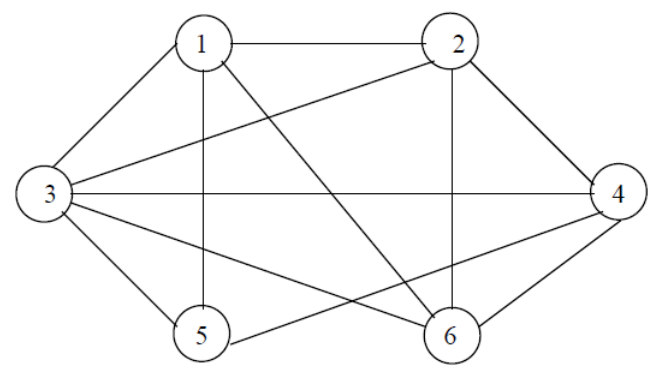

Figure 11 Network N2-2 [68 - Fig. 1]

The third of these networks will be designated as N2-3 and is displayed in figure 12. It is a network with 12 nodes and 50 unidirectional links ( 25 bidirectional links in the original network), with an average node degree of 4.17. This network has also been used in [52].

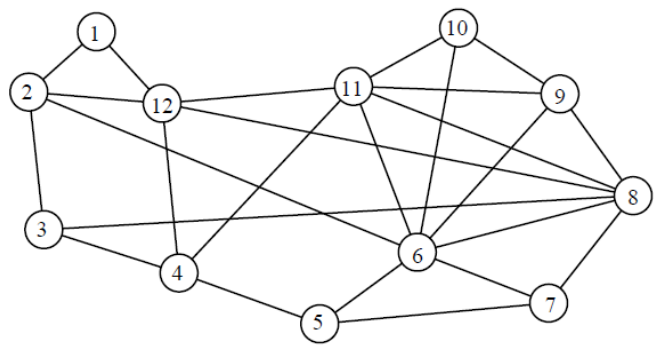

Figure 12 Network N2-3 [68 - Fig. 3]

The last of these networks will be designated as N2-4 and is displayed in figure 13. It is a network with 10 nodes and 56 unidirectional links ( 28 bidirectional links in the original network), with an average node degree of 5.60. This network has also been used in [52].

The third set of networks, designated by N3, is a set of randomly generated MPLS network topologies obtained with the gt-itm software [78], given the desired number of nodes $n$ and the desired average node degree $a$. With the used software, the topology of Internet networks may be randomly generated with different probability laws of occurrence of a link between two nodes. Typically, that probability law is an exponential function of the Euclidian distance between the nodes and of a set of calibration parameters. With these models, there is an attempt to simulate realistic structures of Internet-type networks [24]. 


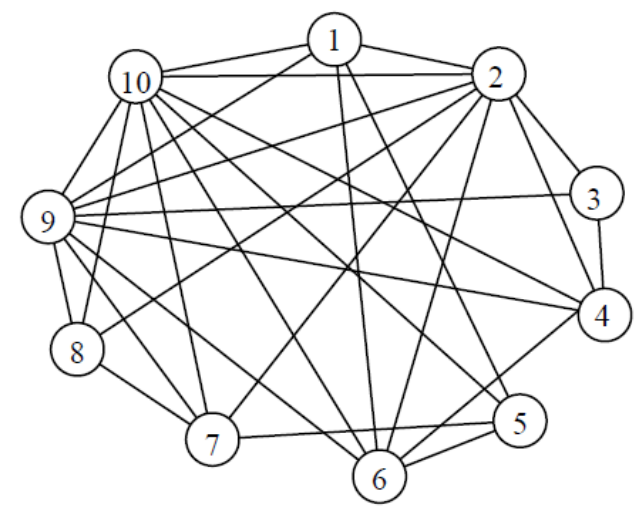

Figure 13 Network N2-4 [68 - Fig. 2]

In this experimental study, and similarly to the study in [16], the model that was considered for the probability distribution of the existence of links was the Doar-Leslie model [24], calibrated so as to guarantee the desired value for the average node degree $a$, considering the stipulated number of nodes $n$. The used software generates information on the nodes which are connected by a pair of unidirectional links.

For each pair of values $(n ; a)$ we have obtained 5 different topologies with an average of $n \cdot a$ unidirectional links. We have considered networks with ( $n=14 ; a=2.7)$, which will be designated as N3$1 ;(n=14 ; a=4.0)$, which will be designated as N3-2; $(n=30 ; a=2.7)$, which will be designated as N3-3; $(n=30 ; a=4.0)$, which will be designated as N3-4.

Notice that in some of the networks in sets N1 and N2 which we considered in this study, the links were originally bidirectional. Instead, we have considered the links to be unidirectional with capacity equal to the provided one, i.e. if a bidirectional link between nodes $i$ and $j$ with a capacity $u$ is given in the original network, we have considered the existence of unidirectional links from $i$ to $j$ and from $j$ to $i$, both with capacity $u$. This adaptation has to do with the nature of MPLS networks, requiring a representation with unidirectional links. The same approach was followed for the demand volumes: if a demand of $v$ is offered between nodes $i$ and $j$ in the original network, we have considered the existence of a demand of $v$ from $i$ to $j$ and also a demand of $v$ from $j$ to $i$.

For some of the networks in set N1, the original link capacities provided in the references had to be slightly increased for the resolution of our problem. For instance, for networks N1-1b and N1-3, the link capacities had to be increased by $20 \%$, so that our problem could be solved. For a problem similar to ours, but with only one type of service (i.e. a mono-service formulation) without any constraint on the maximal number of links $D_{s}$ for the service, then the provided capacity would have been adequate.

For the networks in sets N2 and N3, no information on the offered traffic and link capacities is provided in the original papers. In this situation, we have followed the dimensioning criteria in [68]. A 
fixed value of $100 \mathrm{Mbit} / \mathrm{s}$ is assumed to be the demand volume between all pairs of nodes. The capacity of the links is calculated so as to guarantee that all the links have less than $67 \%$ utilization when shortest path routing between any pair of nodes is considered. The link capacity obtained with this very simple procedure is called the baseline capacity.

For the randomly generated networks, information on the lengths of the links is provided by the used software for a given maximal length of $35 \mathrm{~km}$, typical of metropolitan type networks, as in [14]. For the other networks (in sets N1 and N2), we have devised a simple method to estimate the lengths of the links in each network. We have superimposed each network on a rectangular grid with $400 * 240$ points where the mesh space unit corresponds to $10 \mathrm{~km}$, as in [10]. Therefore, the maximal horizontal distance in the grid is $l_{\max }=4000 \mathrm{~km}$. With this value as reference, we have obtained the length of each of the links $l_{k}, k \in A$ in the networks. This technique was used for all the networks for which the lengths of the links were not provided in the original papers they appear in, i.e. for networks in sets N1 and N2.

\subsection{Service Description}

A total of $S=4$ services were considered. In table 1 , the values of $q_{s}$, the fraction of bandwidth associated with each service, are given.

Table 1 Features of the services in the network tests

\begin{tabular}{cc}
\hline Service & $q_{s}$ \\
\hline 0 - video (QoS) & 0.1 \\
1 - Premium data (QoS) & 0.25 \\
2 - voice (QoS) & 0.4 \\
3 - data (BE) & 0.25 \\
\hline
\end{tabular}

\subsection{Tests Description}

The test environment that we considered in our study considers the type of tests used in [68]. For each network we performed 4 different types of tests.

In test $\mathrm{T} 1$, the offered traffic matrix is fixed, either with a value provided in $[27,54,59]$ (for N1 networks) or with the value of $100 \mathrm{Mbit} / \mathrm{s}$ (for N2 and N3 networks); the link capacities are the so-called baseline capacities, i.e. the ones provided in literature (for N1 networks) or with the value calculated so as to guarantee a maximal link utilization of $67 \%$ (for N2 and N3 networks), as explained earlier.

In test $\mathrm{T} 2$, the offered traffic matrix is randomly generated, with a uniform distribution (as in [52]) using as the average demand volume for each demand pair the value provided in [27, 54, 59] (for N1 networks) or the value of $100 \mathrm{Mbit} / \mathrm{s}$ (for N2 and N3 networks). A total of 16 instances are considered, as in [52], with different seeds allowing for the generation of different traffic matrices. This number of instances guarantees the statistical relevance of the obtained results. The link capacities are the baseline capacities.

In test $\mathrm{T} 3$, the offered traffic matrix is fixed as in $\mathrm{T} 1$, but in this case the networks have a $50 \%$ 
additional capacity derived by multiplying the baseline capacity of each link by 1.5 .

In test $\mathrm{T} 4$, the offered traffic matrix is randomly generated as in $\mathrm{T} 2$, but in this case the networks have a $50 \%$ additional capacity derived by multiplying the baseline capacity of each link by 1.5 . A total of 16 instances of the offered traffic matrix are considered.

Note that for the randomly generated networks (N3), a double randomness exists in tests T2 and T4, as not only the MPLS networks are randomly generated but also the matrix of the traffic offered to those networks is randomly generated.

The cost of each link is given by (17), with $\alpha=0.1$ and $\beta=1-\alpha=0.9$.

In the different formulations of the problem, it is necessary to define a value for the maximal number of paths used by each flow, $N_{L}$, and in this experimental study it was equal to 4 .

For the execution of the algorithms $M C C$ and $M C M$, we have considered $\Delta=10$.

\subsection{Network Performance Measures}

Using the described networks an extensive number of experiments were carried out enabling the calculation of the values of network performance measures, relevant from a teletraffic engineering point of view, for the routing solutions obtained with the algorithms. Some of these performance parameters are 'standard' measures of network performance often used in the evaluation of routing models, namely those based in single criterion optimization approaches such as the one in [68]. This is the case of the parameters total fraction of used capacity, sum of the link utilization and the maximal link utilization.

For each problem (i.e. a specific network structure with a specific traffic matrix), 4 different solutions are obtained: $S_{1}$, the solution obtained when only the objective function $F_{1}$ is minimized; $S_{2}$, the solution obtained when only the objective function $F_{2}$ is minimized; $S_{M C C}$, the solution obtained when the algorithm based on the constraint method (see sub-section 3.1) is used to solve the multiobjective problem; $S_{M C M}$, the solution obtained when the algorithm based on the MCM (see subsection 3.2) is used to solve the multiobjective problem.

The following performance measures were defined:

- total fraction of used capacity: $F U C=\frac{\sum_{k \in A} f_{k}}{\sum_{k \in A} u_{k}}$;

- $\quad$ sum of the link utilization: $S L U=\sum_{k \in A} \frac{f_{k}}{u_{k}}$;

- $\quad$ maximal link utilization: $M L U=\max _{k \in A}\left\{\frac{f_{k}}{u_{k}}\right\}$;

- relative variation with respect to the marginal optima, given by $R V_{\zeta}=\left|\frac{F_{\zeta}^{\text {sol }}-F_{\zeta}^{\text {opt }}}{F_{\zeta}^{\text {opt }}}\right|$ (with $\zeta=1,2$ ) and where $F_{\zeta}^{\text {sol }}$ is the value of $F_{\zeta}$ calculated for a specific multiobjective solution and $F_{\zeta}^{\text {opt }}$ is the optimal value of $F_{\zeta}$ for the same problem.

- time of execution of the algorithms using CPLEX 12.3 in a laptop computer with i7 processor, 2.2 GHz clock and $1 \mathrm{~GB}$ of RAM, running on a Linux virtual machine over the Windows operating system. 
For networks N1 and N2, we obtained the value of the network performance measures when test T1 was executed (i.e. the routing problem is considered with a fixed traffic matrix and the baseline link capacities) and then we ran 16 instances of test T2 (i.e. the routing problem is considered with a random traffic matrix keeping the baseline link capacities). For these 16 instances, we considered all the results and calculated the minimum, the average and the maximal value of each network performance measure. Similarly, we ran test T3 and 16 instances of test T4, obtaining again the values of the network performance measures for the fixed traffic matrix (T3), and the minimum, the average and the maximal value of each network performance measure for the random traffic matrices (T4).

For networks N3, we proceeded likewise, but instead of displaying the results when test T1 (or T3) was executed for a specific network, we considered the obtained values for the 5 different topologies and estimated their mean value and their variation range. Considering the value of a performance measure for the $z$-th topology as $M_{z}$, with $z=0, \ldots, 4$, the estimate of the mean is $\hat{M}$ and the estimate of the variation range is $\frac{2.776}{\sqrt{5}} \hat{\sigma}(\hat{M})$, calculated as a $95 \%$ confidence interval with a t-Student bi-lateral distribution, where $\hat{\sigma}(\hat{M})$ is an estimate of a standard deviation for $M$.

For the minimum, the average and the maximal value of each network performance measure obtained when test T2 (or T4) was executed for each of the 5 different topologies, we also estimated a mean value and a variation range in the same manner.

\subsection{Results}

Results for the different tests (T1 to T4) for networks N1-1b, N1-3, N1-4, N2-3, N3-1, N3-2 and N34 are shown in tables 2-8. The results for the other networks are omitted here, but can be seen in the research report [35] and follow the same qualitative trends.

The values of the objective functions and of the network performance measures are displayed for the solutions of single objective problems (when only $F_{1}$ was optimized, situation $S_{1}$, and only $F_{2}$ was optimized, situation $S_{2}$ ) and bi-objective problems (when $M C C$ was used, situation $S_{M C C}$, and $M C M$ was used, situation $S_{M C M}$ ). We did not display the execution time of $S_{1}$ and $S_{2}$ because these single objective problems were not solved in an isolated manner, but rather at the beginning of the execution of $M C C$ and $M C M$.

These results confirm that the objective functions $F_{1}$ and $F_{2}$ are indeed conflicting, as the minimization of one of them entails an increase in the value of the other objective function.

When we optimize only the objective function $F_{1}$ (results identified by $S_{1}$ in the tables) we realize that the total cost of carrying the bandwidth of all the flows is indeed the lowest, but that is accompanied by a noticeable increase in the utilization of the links in almost all the tested networks, as the values of $F U C, S L U$ and $M L U$ tend to be higher than when only the objective function $F_{2}$ is optimized (results identified by $S_{2}$ in the tables) or when the multiobjective problem is considered (results identified by $S_{M C C}$ or $S_{M C M}$ in the tables, according to the used method of resolution).

When we optimize only the objective function $F_{2}$, the cost of carrying the bandwidth of all the flows 
greatly increases, as it can be seen by analyzing the value of $F_{1}$. The utilization of the links (measured in different forms) is lower in almost all the situations in the tested networks, which makes sense as the minimization of the function $F_{2}$ tends to minimize the global utilization of the links. The decrease in the utilization of the links can be confirmed not only by the lower value of $F_{2}$ but also by the lower values of the performance measures $F U C, S L U$ (in almost all the cases) and $M L U$ (in all the cases). In particular, the $M L U$ is a relevant measurement in traffic engineering terms and its behavior is in all experiments congruent with the function $F_{2}$. The solutions $S_{M C C}$ and $S_{M C M}$ lead to compromise values of $M L U$, excepting when one of the links is fully occupied in the obtained solutions $(M L U=1)$. Note that when $F_{1}$ is optimized, in almost all cases $M L U=1$, which reflects the clear conflicting nature between $F_{1}$ and $F_{2} / M L U$, as noted in earlier studies in single criterion network flow models, as in [68].

Concerning the results for the networks with additional link capacity, the values for $F_{1}$ and $F_{2}$ obtained for solutions $S_{1}, S_{2}, S_{M C C}$ and $S_{M C M}$ are always lower than for the baseline link capacity corresponding cases, as expected taking into account the nature of the objective functions.

In these experiments, we could confirm the potential advantages of using a multiobjective optimization model, rather than a single objective one. In fact, the advantages of using two objectives in this routing problem become clear, as we get a compromise solution that tries to balance the cost of carrying the bandwidth and the global effect of the utilization of the links.

The solutions obtained with the $M C C$ and $M C M$ algorithms may be compared. In both cases, the relative variation of the obtained solutions is higher for $F_{2}$ than for $F_{1}$.

To illustrate the differences between the results obtained with the two algorithms, we present in figure 14 the sets of solutions obtained for both algorithms (in the cases of baseline link capacity (increased by $20 \%$ ) and $50 \%$ additional link capacity) for network N1-1b. In these figures, the solutions obtained in both runs of the algorithms are displayed, where the first run corresponds to the situation where the feasible region is limited by points $X$ (the solution $\left(F_{1}, F_{2}\right) \equiv\left(F_{1}^{\min }, F_{2}^{\max }\right)$ ) and $Y$ (the solution $\left.\left(F_{1}, F_{2}\right) \equiv\left(F_{1}^{\max }, F_{2}^{\min }\right)\right)$ and the second run corresponds to the situation where a specific region of the Pareto front is more thoroughly analyzed. The values of $F_{\zeta}^{r e q}$ and $F_{\zeta}^{a c}$, with $\zeta=1,2$, are obtained in step 5 of each algorithm. The value of the selected Pareto optimal solution (i.e. the final solution provided by each algorithm), obtained in step 7 of each algorithm, is also displayed in the figures.

From figure 14, it is noticeable that the $M C M$ algorithm provides more evenly distributed solutions throughout the Pareto front than the $M C C$ algorithm, both in the first run and in the second run of the algorithms. Considering the displayed results, we can see that in some cases (figures 14c and 14d) the specific region of the Pareto front that is more thoroughly analyzed in the second run of the algorithms is $A$ (the requested levels are satisfied for both objective functions), while in other cases (figures 14a and 14b) the specific region of the Pareto front that is more thoroughly analyzed in the second run of the algorithms is $B_{1}$ (only the requested value for $F_{1}$ is satisfied and an acceptable value is guaranteed for $F_{2}$ ). As for the final solution obtained with the algorithms, notice that sometimes it was obtained in the first run of the algorithm (see figure 14b), while in the other cases it was obtained in the second run 
Table 2 Network performance measure values, for network N1-1b

\begin{tabular}{|c|c|c|c|c|c|c|c|c|c|}
\hline \multirow{3}{*}{\multicolumn{2}{|c|}{$\begin{array}{l}\text { Network } \\
\text { performance Method } \\
\text { measure }\end{array}$}} & \multicolumn{4}{|c|}{ Baseline capacity (increased by $20 \%$ ) } & \multicolumn{4}{|c|}{$50 \%$ additional capacity } \\
\hline & & \multirow{2}{*}{$\begin{array}{l}\text { Fixed } \\
\text { traffic }\end{array}$} & \multicolumn{3}{|c|}{ Random traffic matrix } & \multirow{2}{*}{$\begin{array}{l}\text { Fixed } \\
\text { traffic }\end{array}$} & \multicolumn{3}{|c|}{ Random traffic matrix } \\
\hline & & & $\min$ & avg & $\max$ & & $\min$ & avg & $\max$ \\
\hline \multirow{4}{*}{$F_{1}$} & $S_{1}$ & 2189.42 & 1905.06 & 2142.40 & 2336.84 & 2136.11 & 1835.85 & 2046.36 & 2202.95 \\
\hline & $S_{2}$ & 2337.40 & 2077.81 & 2295.26 & 2447.50 & 2342.44 & 2077.05 & 2287.06 & 2437.74 \\
\hline & $S_{M C C}$ & 2212.70 & 1949.50 & 2170.96 & 2355.43 & 2170.01 & 1888.65 & 2090.22 & 2244.13 \\
\hline & $S_{M C M}$ & 2211.94 & 1950.13 & 2171.44 & 2355.76 & 2170.12 & 1885.70 & 2090.46 & 2245.94 \\
\hline \multirow{4}{*}{$F_{2}$} & $S_{1}$ & 146072.21 & 89714.32 & 141932.66 & 187991.45 & 59167.12 & 34362.59 & 60954.54 & 99224.36 \\
\hline & $S_{2}$ & 46071.52 & 22850.45 & 67416.15 & 119179.28 & 11741.40 & 8611.40 & 12681.67 & 16667.83 \\
\hline & $S_{M C C}$ & 93789.25 & 40523.62 & 99587.39 & 156604.00 & 19317.79 & 12565.17 & 21792.40 & 32071.40 \\
\hline & $S_{M C M}$ & 94661.03 & 40251.29 & 99234.74 & 156310.33 & 19300.65 & 12463.58 & 21734.82 & 31704.86 \\
\hline \multirow{3}{*}{$R V_{1}$} & $S_{2}$ & $6.76 \%$ & $2.62 \%$ & $7.28 \%$ & $12.15 \%$ & $9.66 \%$ & $9.90 \%$ & $11.83 \%$ & $14.84 \%$ \\
\hline & $S_{M C C}$ & $1.06 \%$ & $0.48 \%$ & $1.38 \%$ & $2.92 \%$ & $1.59 \%$ & $1.61 \%$ & $2.15 \%$ & $2.89 \%$ \\
\hline & $S_{M C M}$ & $1.03 \%$ & $0.48 \%$ & $1.40 \%$ & $2.91 \%$ & $1.59 \%$ & $1.66 \%$ & $2.16 \%$ & $2.88 \%$ \\
\hline \multirow{3}{*}{$R V_{2}$} & $S_{1}$ & $217.06 \%$ & $16.30 \%$ & $156.73 \%$ & $403.04 \%$ & $403.92 \%$ & $237.68 \%$ & $376.54 \%$ & $599.09 \%$ \\
\hline & $S_{M C C}$ & $103.57 \%$ & $9.26 \%$ & $58.10 \%$ & $98.36 \%$ & $64.53 \%$ & $40.96 \%$ & $70.26 \%$ & $123.57 \%$ \\
\hline & $S_{M C M}$ & $105.47 \%$ & $9.32 \%$ & $57.37 \%$ & $98.39 \%$ & $64.38 \%$ & $40.05 \%$ & $70.01 \%$ & $121.06 \%$ \\
\hline \multirow{4}{*}{ FUC } & $S_{1}$ & 0.8461 & 0.7496 & 0.8168 & 0.8720 & 0.6886 & 0.6038 & 0.6601 & 0.7136 \\
\hline & $S_{2}$ & 0.8361 & 0.7452 & 0.8141 & 0.8712 & 0.6694 & 0.5958 & 0.6498 & 0.6960 \\
\hline & $S_{M C C}$ & 0.8358 & 0.7411 & 0.8113 & 0.8692 & 0.6728 & 0.5953 & 0.6500 & 0.6987 \\
\hline & $S_{M C M}$ & 0.8361 & 0.7411 & 0.8113 & 0.8693 & 0.6728 & 0.5953 & 0.6498 & 0.6986 \\
\hline \multirow{4}{*}{$S L U$} & $S_{1}$ & 30.4235 & 27.5088 & 29.5296 & 31.4678 & 25.8608 & 23.2842 & 24.7494 & 26.3017 \\
\hline & $S_{2}$ & 30.2821 & 27.1343 & 29.7031 & 31.8564 & 24.1047 & 21.6867 & 23.7326 & 25.3974 \\
\hline & $S_{M C C}$ & 30.2201 & 26.8670 & 29.3908 & 31.6265 & 24.5549 & 22.0766 & 23.7395 & 25.3237 \\
\hline & $S_{M C M}$ & 30.2172 & 26.8651 & 29.3938 & 31.6281 & 24.5554 & 22.0671 & 23.7436 & 25.3306 \\
\hline \multirow{4}{*}{$M L U$} & $S_{1}$ & 1.0000 & 1.0000 & 1.0000 & 1.0000 & 1.0000 & 1.0000 & 1.0000 & 1.0000 \\
\hline & $S_{2}$ & 0.9000 & 0.9000 & 0.9748 & 1.0000 & 0.7000 & 0.7000 & 0.7822 & 0.8000 \\
\hline & $S_{M C C}$ & 1.0000 & 1.0000 & 1.0000 & 1.0000 & 0.9000 & 0.9000 & 0.9009 & 0.9098 \\
\hline & $S_{M C M}$ & 1.0000 & 1.0000 & 1.0000 & 1.0000 & 0.9000 & 0.9000 & 0.9010 & 0.9099 \\
\hline \multirow{2}{*}{ Time [s] } & $S_{M C C}$ & 4.52 & 4.31 & 4.68 & 5.04 & 4.15 & 3.95 & 4.20 & 4.51 \\
\hline & $S_{M C M}$ & 4.79 & 4.37 & 4.87 & 5.41 & 4.51 & 4.25 & 4.52 & 4.88 \\
\hline
\end{tabular}


Table 3 Network performance measure values, for network N1-3

\begin{tabular}{|c|c|c|c|c|c|c|c|c|c|}
\hline \multirow{3}{*}{\multicolumn{2}{|c|}{$\begin{array}{l}\text { Network } \\
\text { performance Method } \\
\text { measure }\end{array}$}} & \multicolumn{4}{|c|}{ Baseline capacity (increased by $20 \%$ ) } & \multicolumn{4}{|c|}{$50 \%$ additional capacity } \\
\hline & & \multirow{2}{*}{$\begin{array}{l}\text { Fixed } \\
\text { traffic }\end{array}$} & \multicolumn{3}{|c|}{ Random traffic matrix } & \multirow{2}{*}{$\begin{array}{l}\text { Fixed } \\
\text { traffic }\end{array}$} & \multicolumn{3}{|c|}{ Random traffic matrix } \\
\hline & & & $\min$ & avg & $\max$ & & $\min$ & avg & $\max$ \\
\hline \multirow{4}{*}{$F_{1}$} & $S_{1}$ & 1652.05 & 1155.34 & 1415.05 & 1655.22 & 1573.82 & 1125.00 & 1358.55 & 1566.63 \\
\hline & $S_{2}$ & 1749.76 & 1275.48 & 1516.04 & 1761.56 & 1698.45 & 1269.60 & 1498.09 & 1743.35 \\
\hline & $S_{M C C}$ & 1670.94 & 1177.31 & 1433.72 & 1669.57 & 1603.71 & 1149.66 & 1386.53 & 1602.89 \\
\hline & $S_{M C M}$ & 1670.84 & 1177.60 & 1433.71 & 1669.49 & 1603.71 & 1148.44 & 1386.43 & 1602.27 \\
\hline \multirow{4}{*}{$F_{2}$} & $\overline{S_{1}}$ & 37657.93 & 17529.72 & 29494.54 & 48869.50 & 36830.87 & 12556.25 & 22777.95 & 33601.79 \\
\hline & $S_{2}$ & 21759.40 & 2835.42 & 12126.90 & 28784.50 & 4438.20 & 2258.04 & 3357.99 & 4651.24 \\
\hline & $S_{M C C}$ & 24538.16 & 6872.48 & 16770.74 & 33457.21 & 12228.77 & 4254.23 & 7335.75 & 10447.51 \\
\hline & $S_{M C M}$ & 24578.17 & 6823.51 & 16787.74 & 33476.28 & 12229.26 & 4266.41 & 7355.98 & 10672.46 \\
\hline \multirow{3}{*}{$R V_{1}$} & $S_{2}$ & $5.91 \%$ & $4.15 \%$ & $7.29 \%$ & $11.04 \%$ & $7.92 \%$ & $6.34 \%$ & $10.40 \%$ & $15.57 \%$ \\
\hline & $S_{M C C}$ & $1.14 \%$ & $0.73 \%$ & $1.36 \%$ & $2.35 \%$ & $1.90 \%$ & $1.38 \%$ & $2.07 \%$ & $2.99 \%$ \\
\hline & $S_{M C M}$ & $1.14 \%$ & $0.72 \%$ & $1.35 \%$ & $2.36 \%$ & $1.90 \%$ & $1.41 \%$ & $2.06 \%$ & $2.99 \%$ \\
\hline \multirow{3}{*}{$R V_{2}$} & $S_{1}$ & $73.07 \%$ & $35.76 \%$ & $267.90 \%$ & $805.30 \%$ & $729.86 \%$ & $383.40 \%$ & $583.22 \%$ & $813.98 \%$ \\
\hline & $S_{M C C}$ & $12.77 \%$ & $5.51 \%$ & $69.06 \%$ & $186.85 \%$ & $175.53 \%$ & $79.18 \%$ & $117.29 \%$ & $193.33 \%$ \\
\hline & $S_{M C M}$ & $12.95 \%$ & $5.54 \%$ & $69.20 \%$ & $192.27 \%$ & $175.55 \%$ & $79.70 \%$ & $117.83 \%$ & $193.34 \%$ \\
\hline \multirow{4}{*}{$F U C$} & $S_{1}$ & 0.6574 & 0.4937 & 0.5845 & 0.6691 & 0.5132 & 0.4006 & 0.4683 & 0.5269 \\
\hline & $S_{2}$ & 0.6646 & 0.4920 & 0.5832 & 0.6739 & 0.5262 & 0.3889 & 0.4622 & 0.5362 \\
\hline & $S_{M C C}$ & 0.6648 & 0.4887 & 0.5860 & 0.6691 & 0.5194 & 0.3961 & 0.4674 & 0.5322 \\
\hline & $S_{M C M}$ & 0.6648 & 0.4883 & 0.5860 & 0.6692 & 0.5194 & 0.3961 & 0.4673 & 0.5321 \\
\hline \multirow{4}{*}{$S L U$} & $S_{1}$ & 13.4712 & 10.2263 & 12.0008 & 13.6988 & 10.4723 & 8.3167 & 9.6173 & 10.8271 \\
\hline & $S_{2}$ & 13.6438 & 10.1836 & 11.9630 & 13.6882 & 10.7826 & 7.9727 & 9.4682 & 10.8806 \\
\hline & $S_{M C C}$ & 13.6476 & 10.1054 & 12.0377 & 13.7306 & 10.6200 & 8.2083 & 9.5946 & 10.8597 \\
\hline & $S_{M C M}$ & 13.6476 & 10.0962 & 12.0362 & 13.7352 & 10.6200 & 8.2083 & 9.5942 & 10.8584 \\
\hline \multirow{4}{*}{$M L U$} & $S_{1}$ & 1.0000 & 1.0000 & 1.0000 & 1.0000 & 1.0000 & 1.0000 & 1.0000 & 1.0000 \\
\hline & $S_{2}$ & 1.0000 & 0.8000 & 0.9140 & 1.0000 & 0.8000 & 0.6051 & 0.7341 & 0.8000 \\
\hline & $S_{M C C}$ & 1.0000 & 0.9000 & 0.9684 & 1.0000 & 0.9268 & 0.8482 & 0.8976 & 0.9246 \\
\hline & $S_{M C M}$ & 1.0000 & 0.9000 & 0.9686 & 1.0000 & 0.9268 & 0.8562 & 0.8987 & 0.9247 \\
\hline \multirow{2}{*}{ Time [s] } & $S_{M C C}$ & 0.83 & 0.75 & 0.80 & 0.85 & 0.78 & 0.72 & 0.76 & 0.83 \\
\hline & $S_{M C M}$ & 0.90 & 0.77 & 0.82 & 0.86 & 0.83 & 0.76 & 0.79 & 0.82 \\
\hline
\end{tabular}


Table 4 Network performance measure values, for network N1-4

\begin{tabular}{|c|c|c|c|c|c|c|c|c|c|}
\hline \multirow{3}{*}{\multicolumn{2}{|c|}{$\begin{array}{l}\text { Network } \\
\text { performance Method } \\
\text { measure } \\
\end{array}$}} & \multicolumn{4}{|c|}{ Baseline capacity } & \multicolumn{4}{|c|}{$50 \%$ additional capacity } \\
\hline & & \multirow{2}{*}{$\begin{array}{l}\text { Fixed } \\
\text { traffic }\end{array}$} & \multicolumn{3}{|c|}{ Random traffic matrix } & \multirow{2}{*}{$\begin{array}{l}\text { Fixed } \\
\text { traffic }\end{array}$} & \multicolumn{3}{|c|}{ Random traffic matrix } \\
\hline & & & $\min$ & avg & $\max$ & & $\min$ & avg & $\max$ \\
\hline \multirow{4}{*}{$F_{1}$} & $S_{1}$ & 368.73 & 306.27 & 356.03 & 403.78 & 368.73 & 306.27 & 354.76 & 400.38 \\
\hline & $S_{2}$ & 415.51 & 346.27 & 405.34 & 456.35 & 411.16 & 342.75 & 402.42 & 457.60 \\
\hline & $S_{M C C}$ & 378.08 & 311.67 & 364.62 & 413.92 & 375.46 & 311.78 & 362.89 & 407.71 \\
\hline & $S_{M C M}$ & 377.88 & 311.71 & 364.45 & 414.13 & 375.96 & 312.07 & 362.69 & 408.20 \\
\hline \multirow{4}{*}{$F_{2}$} & $S_{1}$ & 5913.53 & 3237.97 & 8991.79 & 14133.26 & 1032.00 & 842.55 & 1104.57 & 1335.30 \\
\hline & $S_{2}$ & 1082.00 & 761.83 & 1178.11 & 1597.02 & 845.90 & 706.53 & 824.72 & 922.55 \\
\hline & $S_{M C C}$ & 2017.48 & 1094.32 & 2558.97 & 4100.60 & 943.36 & 771.65 & 931.11 & 1106.67 \\
\hline & $S_{M C M}$ & 2042.87 & 1092.59 & 2587.92 & 4041.17 & 939.56 & 770.36 & 933.31 & 1099.08 \\
\hline \multirow{3}{*}{$R V_{1}$} & $S_{2}$ & $12.69 \%$ & $9.81 \%$ & $13.99 \%$ & $20.33 \%$ & $11.51 \%$ & $9.07 \%$ & $13.56 \%$ & $21.44 \%$ \\
\hline & $S_{M C C}$ & $2.54 \%$ & $1.36 \%$ & $2.42 \%$ & $3.47 \%$ & $1.82 \%$ & $1.45 \%$ & $2.33 \%$ & $5.24 \%$ \\
\hline & $S_{M C M}$ & $2.48 \%$ & $1.39 \%$ & $2.37 \%$ & $3.35 \%$ & $1.96 \%$ & $1.27 \%$ & $2.27 \%$ & $5.24 \%$ \\
\hline \multirow{3}{*}{$R V_{2}$} & $S_{1}$ & $446.54 \%$ & $325.03 \%$ & $652.46 \%$ & $785.33 \%$ & $22.00 \%$ & $19.25 \%$ & $33.71 \%$ & $56.53 \%$ \\
\hline & $S_{M C C}$ & $86.46 \%$ & $43.64 \%$ & $113.76 \%$ & $156.76 \%$ & $11.52 \%$ & $6.91 \%$ & $12.75 \%$ & $19.96 \%$ \\
\hline & $S_{M C M}$ & $88.80 \%$ & $43.42 \%$ & $116.28 \%$ & $153.04 \%$ & $11.07 \%$ & $6.99 \%$ & $13.01 \%$ & $19.14 \%$ \\
\hline \multirow{4}{*}{ FUC } & $S_{1}$ & 0.5928 & 0.5030 & 0.5724 & 0.6386 & 0.3952 & 0.3353 & 0.3828 & 0.4295 \\
\hline & $S_{2}$ & 0.5391 & 0.4473 & 0.5259 & 0.5833 & 0.3525 & 0.2944 & 0.3427 & 0.3819 \\
\hline & $S_{M C C}$ & 0.5752 & 0.4890 & 0.5586 & 0.6144 & 0.3777 & 0.3194 & 0.3687 & 0.4159 \\
\hline & $S_{M C M}$ & 0.5755 & 0.4887 & 0.5588 & 0.6139 & 0.3769 & 0.3189 & 0.3688 & 0.4144 \\
\hline \multirow{4}{*}{$S L U$} & $S_{1}$ & 18.9710 & 16.0944 & 18.3182 & 20.4365 & 12.6473 & 10.7296 & 12.2510 & 13.7441 \\
\hline & $S_{2}$ & 17.2500 & 14.3122 & 16.8301 & 18.6664 & 11.2787 & 9.4203 & 10.9663 & 12.2222 \\
\hline & $S_{M C C}$ & 18.4073 & 15.6468 & 17.8763 & 19.6605 & 12.0851 & 10.2216 & 11.7995 & 13.3101 \\
\hline & $S_{M C M}$ & 18.4146 & 15.6398 & 17.8815 & 19.6444 & 12.0597 & 10.2044 & 11.8019 & 13.2595 \\
\hline \multirow{4}{*}{$M L U$} & $S_{1}$ & 0.9960 & 0.9715 & 0.9965 & 1.0000 & 0.6640 & 0.6477 & 0.7305 & 0.8262 \\
\hline & $S_{2}$ & 0.7000 & 0.6413 & 0.7566 & 0.8000 & 0.5000 & 0.5000 & 0.5206 & 0.5729 \\
\hline & $S_{M C C}$ & 0.8000 & 0.7494 & 0.8832 & 0.9041 & 0.6000 & 0.5670 & 0.6164 & 0.7000 \\
\hline & $S_{M C M}$ & 0.8063 & 0.7494 & 0.8868 & 0.9067 & 0.6000 & 0.5670 & 0.6212 & 0.7000 \\
\hline \multirow{2}{*}{ Time [s] } & $S_{M C C}$ & 2.08 & 1.92 & 2.13 & 2.28 & 1.79 & 1.69 & 1.88 & 2.05 \\
\hline & $S_{M C M}$ & 2.09 & 1.95 & 2.08 & 2.24 & 1.74 & 1.69 & 1.80 & 1.91 \\
\hline
\end{tabular}


Table 5 Network performance measure values, for network N2-3

\begin{tabular}{|c|c|c|c|c|c|c|c|c|c|}
\hline \multirow{3}{*}{\multicolumn{2}{|c|}{$\begin{array}{l}\text { Network } \\
\text { performance Method } \\
\text { measure }\end{array}$}} & \multirow{2}{*}{\multicolumn{4}{|c|}{ Baseline capacity }} & \multicolumn{4}{|c|}{$50 \%$ additional capacity } \\
\hline & & & & & & \multirow{2}{*}{$\begin{array}{l}\text { Fixed } \\
\text { traffic }\end{array}$} & \multicolumn{3}{|c|}{ Random traffic matrix } \\
\hline & & $\begin{array}{l}\text { Fixed } \\
\text { traffic }\end{array}$ & $\min$ & avg & $\max$ & & $\min$ & avg & $\max$ \\
\hline \multirow{4}{*}{$F_{1}$} & $S_{1}$ & 4485.41 & 4026.78 & 4367.52 & 4793.92 & 4153.83 & 3731.93 & 4017.56 & 4340.53 \\
\hline & $S_{2}$ & 5465.25 & 5091.97 & 5486.66 & 5976.78 & 5215.98 & 4734.19 & 5207.97 & 5813.52 \\
\hline & $S_{M C C}$ & 4659.88 & 4232.35 & 4559.10 & 5024.91 & 4271.85 & 3860.11 & 4144.08 & 4504.19 \\
\hline & $S_{M C M}$ & 4651.44 & 4237.74 & 4560.00 & 5017.63 & 4263.50 & 3844.93 & 4141.62 & 4497.91 \\
\hline \multirow{4}{*}{$F_{2}$} & $S_{1}$ & 557563.01 & 358541.73 & 482654.89 & 618306.77 & 315425.00 & 176049.24 & 306398.32 & 429400.15 \\
\hline & $S_{2}$ & 34720.00 & 27389.29 & 40245.73 & 53367.07 & 22400.00 & 20065.09 & 21864.26 & 23338.37 \\
\hline & $S_{M C C}$ & 117173.10 & 76888.26 & 116262.28 & 154143.26 & 52474.96 & 37752.17 & 52561.82 & 79012.12 \\
\hline & $S_{M C M}$ & 123573.58 & 80149.05 & 115569.39 & 149706.58 & 55207.50 & 38026.84 & 53557.20 & 78475.58 \\
\hline \multirow{3}{*}{$R V_{1}$} & $S_{2}$ & $21.85 \%$ & $17.34 \%$ & $25.70 \%$ & $35.86 \%$ & $25.57 \%$ & $23.56 \%$ & $29.62 \%$ & $36.43 \%$ \\
\hline & $S_{M C C}$ & $3.89 \%$ & $2.44 \%$ & $4.39 \%$ & $5.56 \%$ & $2.84 \%$ & $2.13 \%$ & $3.15 \%$ & $4.06 \%$ \\
\hline & $S_{M C M}$ & $3.70 \%$ & $2.32 \%$ & $4.41 \%$ & $5.60 \%$ & $2.64 \%$ & $2.04 \%$ & $3.08 \%$ & $4.08 \%$ \\
\hline \multirow{3}{*}{$R V_{2}$} & $S_{1}$ & $1505.88 \%$ & $804.69 \%$ & $1111.52 \%$ & $1371.91 \%$ & $1308.15 \%$ & $734.54 \%$ & $1296.81 \%$ & $1798.79 \%$ \\
\hline & $S_{M C C}$ & $237.48 \%$ & $122.91 \%$ & $189.88 \%$ & $234.88 \%$ & $134.26 \%$ & $76.79 \%$ & $139.70 \%$ & $249.39 \%$ \\
\hline & $S_{M C M}$ & $255.91 \%$ & $132.37 \%$ & $188.38 \%$ & $225.70 \%$ & $146.46 \%$ & $80.26 \%$ & $144.32 \%$ & $247.01 \%$ \\
\hline \multirow{4}{*}{ FUC } & $S_{1}$ & 0.7321 & 0.6503 & 0.7005 & 0.7400 & 0.5228 & 0.4683 & 0.5031 & 0.5307 \\
\hline & $S_{2}$ & 0.6667 & 0.6026 & 0.6551 & 0.6902 & 0.4444 & 0.3942 & 0.4279 & 0.4511 \\
\hline & $S_{M C C}$ & 0.7033 & 0.6320 & 0.6787 & 0.7182 & 0.4964 & 0.4458 & 0.4786 & 0.5057 \\
\hline & $S_{M C M}$ & 0.7039 & 0.6322 & 0.6786 & 0.7180 & 0.4975 & 0.4465 & 0.4788 & 0.5053 \\
\hline \multirow{4}{*}{$S L U$} & $S_{1}$ & 38.2524 & 35.3417 & 36.9044 & 38.7463 & 28.7030 & 26.4874 & 27.6211 & 29.3604 \\
\hline & $S_{2}$ & 33.5816 & 30.6593 & 32.9378 & 34.5051 & 22.7762 & 20.7644 & 21.9480 & 23.0985 \\
\hline & $S_{M C C}$ & 36.6770 & 33.9419 & 35.4391 & 37.3803 & 26.8771 & 24.7630 & 25.7907 & 27.2809 \\
\hline & $S_{M C M}$ & 36.7093 & 33.9370 & 35.4333 & 37.3835 & 26.9626 & 24.6854 & 25.8169 & 27.1206 \\
\hline \multirow{4}{*}{$M L U$} & $S_{1}$ & 1.0000 & 1.0000 & 1.0000 & 1.0000 & 1.0000 & 1.0000 & 1.0000 & 1.0000 \\
\hline & $S_{2}$ & 0.7000 & 0.7194 & 0.8066 & 0.8800 & 0.5000 & 0.6000 & 0.6000 & 0.6000 \\
\hline & $S_{M C C}$ & 0.9000 & 0.9000 & 0.9144 & 0.9767 & 0.9000 & 0.8000 & 0.8717 & 0.9000 \\
\hline & $S_{M C M}$ & 0.9128 & 0.9000 & 0.9116 & 0.9533 & 0.9000 & 0.8000 & 0.8767 & 0.9000 \\
\hline \multirow{2}{*}{ Time [s] } & $S_{M C C}$ & 5.16 & 4.68 & 5.05 & 5.56 & 3.97 & 3.79 & 3.99 & 4.26 \\
\hline & $S_{M C M}$ & 7.10 & 6.22 & 6.96 & 7.64 & 5.23 & 4.74 & 5.27 & 5.85 \\
\hline
\end{tabular}


Table 6 Network performance measure values with variation ranges, for random networks N3-1

\begin{tabular}{|c|c|c|c|c|c|}
\hline $\begin{array}{l}\text { Network } \\
\text { perf. } \\
\text { measure }\end{array}$ & Method & Fixed traffic & Baseline & $\begin{array}{l}\text { capacity } \\
\text { Random traffic matrix } \\
\text { avg }\end{array}$ & $\max$ \\
\hline \multirow{4}{*}{$F_{1}$} & $S_{1}$ & $18161.88 \pm 6769.71$ & $16150.10 \pm 6197.22$ & $17823.34 \pm 6561.94$ & $19590.36 \pm 7192.63$ \\
\hline & $S_{2}$ & $19125.11 \pm 5852.54$ & $17312.31 \pm 5128.11$ & $18931.98 \pm 5578.05$ & $20680.29 \pm 6193.90$ \\
\hline & $S_{M C C}$ & $18364.45 \pm 6585.04$ & $16360.33 \pm 6000.64$ & $18041.32 \pm 6359.15$ & $19787.83 \pm 7009.22$ \\
\hline & $S_{M C M}$ & $18361.08 \pm 6590.10$ & $16360.29 \pm 6002.21$ & $18040.61 \pm 6360.49$ & $19789.36 \pm 7009.39$ \\
\hline \multirow{4}{*}{$F_{2}$} & $S_{1}$ & $422323.35 \pm 319342.51$ & $283557.21 \pm 183353.59$ & $405797.46 \pm 262696.67$ & $529990.34 \pm 317634.4$ \\
\hline & $S_{2}$ & $74028.00 \pm 18958.51$ & $58087.07 \pm 15406.34$ & $86281.77 \pm 22784.87$ & $133922.69 \pm 34278.52$ \\
\hline & $S_{M C C}$ & $187873.94 \pm 117464.14$ & $99206.38 \pm 26725.19$ & $164631.86 \pm 65518.42$ & $287087.53 \pm 160983.12$ \\
\hline & $S_{M C M}$ & $190180.91 \pm 1$ & $99122.36 \pm 27079.77$ & $165081.99 \pm 66857.83$ & $286926.37 \pm 160837.80$ \\
\hline \multirow{3}{*}{$R V_{1}$} & $S_{2}$ & $6.65 \% \pm 7.22 \%$ & $6.21 \% \pm 6.95 \%$ & $7.74 \% \pm 8.07 \%$ & $10.18 \% \pm 9.35 \%$ \\
\hline & $S_{M C C}$ & $1.38 \% \pm 1.56 \%$ & $1.00 \% \pm 1.12 \%$ & $1.53 \% \pm 1.61 \%$ & $2.07 \% \pm 2.06 \%$ \\
\hline & $S_{M C M}$ & $1.35 \% \pm 1.55 \%$ & $0.95 \% \pm 1.07 \%$ & $1.52 \% \pm 1.60 \%$ & $2.08 \% \pm 2.08 \%$ \\
\hline \multirow{3}{*}{$R V_{2}$} & $S_{1}$ & $537.19 \% \pm 540.07 \%$ & $292.87 \% \pm 290.29 \%$ & $434.86 \% \pm 409.95 \%$ & $606.38 \% \pm 526.82 \%$ \\
\hline & $S_{M C C}$ & $171.76 \% \pm 196.48 \%$ & $62.34 \% \pm 66.14 \%$ & $100.71 \% \pm 101.42 \%$ & $159.60 \% \pm 145.65 \%$ \\
\hline & $S_{M C M}$ & $175.25 \% \pm 203.25 \%$ & $62.77 \% \pm 67.15 \%$ & $101.14 \% \pm 102.62 \%$ & $158.73 \% \pm 145.95 \%$ \\
\hline \multirow{4}{*}{ FUC } & $S_{1}$ & $0.6819 \pm 0.0113$ & $0.6116 \pm 0.0178$ & $0.6658 \pm 0.0097$ & $0.7227 \pm 0.0131$ \\
\hline & $S_{2}$ & $0.6667 \pm 0.0000$ & $0.5991 \pm 0.0102$ & $0.6547 \pm 0.0028$ & $0.7160 \pm 0.0101$ \\
\hline & $S_{M C C}$ & $0.6784 \pm 0.0096$ & $0.6043 \pm 0.0137$ & $0.6601 \pm 0.0072$ & $0.7201 \pm 0.0137$ \\
\hline & $S_{M C M}$ & $0.6785 \pm 0.0098$ & $0.6043 \pm 0.0138$ & $0.6601 \pm 0.0072$ & $0.7201 \pm 0.0137$ \\
\hline \multirow{4}{*}{$S L U$} & $S_{1}$ & $25.9320 \pm 6.7478$ & $23.2919 \pm 6.2768$ & $25.3656 \pm 6.5864$ & $27.4647 \pm 7.1687$ \\
\hline & $S_{2}$ & $25.3595 \pm 6.2$ & $220674+58378$ & $24.9751 \pm 6.2008$ & $27.2931 \pm 6.8016$ \\
\hline & $S_{M C C}$ & $25.6522 \pm 6.4574$ & $22.8933 \pm 6.0269$ & $25.0824 \pm 6.3811$ & $27.2694 \pm 7.0380$ \\
\hline & $S_{M C M}$ & $25.6536 \pm 6.4573$ & $22.8890 \pm 6.0226$ & $25.0805 \pm 6.3853$ & $27.2716 \pm 7.0346$ \\
\hline \multirow{4}{*}{$M L U$} & $S_{1}$ & $0.9474 \pm 0.1461$ & $0.9509 \pm 0.1364$ & $0.9666 \pm 0.0929$ & $0.9926 \pm 0.0205$ \\
\hline & $S_{2}$ & $0.6933 \pm 0.0185$ & $0.7473 \pm 0.0469$ & $0.8278 \pm 0.0227$ & $0.9219 \pm 0.0479$ \\
\hline & $S_{M C C}$ & $0.9082 \pm 0.1368$ & $0.8664 \pm 0.0836$ & $0.9190 \pm 0.0760$ & $0.9739 \pm 0.0462$ \\
\hline & $S_{M C M}$ & $0.9081 \pm 0.1369$ & $0.8664 \pm 0.0836$ & $0.9185 \pm 0.0756$ & $0.9736 \pm 0.0466$ \\
\hline \multirow{2}{*}{ Time $[\mathrm{s}]$} & $S_{M C C}$ & $4.83 \pm 3.03$ & $4.47 \pm 2.77$ & $4.76 \pm 2.98$ & $5.08 \pm 3.27$ \\
\hline & $S_{M C M}$ & $5.59 \pm 3.99$ & $5.20 \pm 3.72$ & $5.61 \pm 4.06$ & $6.08 \pm 4.48$ \\
\hline
\end{tabular}


Table 6 (cont.) Network performance measure values with variation ranges, for random networks N3-1

\begin{tabular}{|c|c|c|c|c|c|}
\hline \multirow{3}{*}{$\begin{array}{c}\text { Network } \\
\text { perf. } \\
\text { measure }\end{array}$} & \multirow{3}{*}{ Method } & \multirow{2}{*}{\multicolumn{4}{|c|}{$50 \%$ additional capacity }} \\
\hline & & & & & \\
\hline & & Hace & $\min$ & avg & $\max$ \\
\hline \multirow{4}{*}{$F_{1}$} & $S_{1}$ & $17956.91 \pm 7000.82$ & $15999.18 \pm 6353.27$ & $17555.86 \pm 6845.06$ & $19149.46 \pm 7669.19$ \\
\hline & $S_{2}$ & $18887.42 \pm 6093.38$ & $16851.74 \pm 5577.56$ & $18614.98 \pm 5852.03$ & $20554.81 \pm 6304.75$ \\
\hline & $S_{M C C}$ & $18112.11 \pm 6889.62$ & $16125.66 \pm 6272.98$ & $17706.18 \pm 6720.86$ & $19356.86 \pm 7500.34$ \\
\hline & $S_{M C M}$ & $18101.70 \pm 6865.54$ & $16111.82 \pm 6253.33$ & $17703.48 \pm 6715.96$ & $19358.21 \pm 7497.21$ \\
\hline \multirow{4}{*}{$F_{2}$} & $S_{1}$ & $152851.72 \pm 133312.99$ & $113042.62 \pm 96922.65$ & $167618.69 \pm 128783.24$ & $244433.11 \pm 211426.81$ \\
\hline & $S_{2}$ & $47760.00 \pm 12231.30$ & $42732.77 \pm 10813.16$ & $47086.19 \pm 12162.30$ & $52167.49 \pm 13569.96$ \\
\hline & $S_{M C C}$ & $64753.07 \pm 21735.69$ & $50952.11 \pm 12373.56$ & $63190.02 \pm 17829.73$ & $77500.00 \pm 22992.32$ \\
\hline & $S_{M C M}$ & $64071.00 \pm 20389.06$ & $51540.15 \pm 14936.08$ & $63070.05 \pm 17651.49$ & $78454.20 \pm 22922.16$ \\
\hline \multirow{3}{*}{$R V_{1}$} & $S_{2}$ & $6.65 \% \pm 7.27 \%$ & $5.80 \% \pm 6.39 \%$ & $7.65 \% \pm 8.12 \%$ & $10.47 \% \pm 10.35 \%$ \\
\hline & $S_{M C C}$ & $1.07 \% \pm 1.18 \%$ & $0.65 \% \pm 0.74 \%$ & $1.06 \% \pm 1.15 \%$ & $1.75 \% \pm 1.66 \%$ \\
\hline & $S_{M C M}$ & $1.03 \% \pm 1.24 \%$ & $0.61 \% \pm 0.68 \%$ & $1.05 \% \pm 1.16 \%$ & $1.63 \% \pm 1.77 \%$ \\
\hline \multirow{3}{*}{$R V_{2}$} & $S_{1}$ & $240.96 \% \pm 344.85 \%$ & $153.30 \% \pm 204.92 \%$ & $278.82 \% \pm 341.96 \%$ & $438.01 \% \pm 517.81 \%$ \\
\hline & $S_{M C C}$ & $37.22 \% \pm 39.00 \%$ & $15.43 \% \pm 17.12 \%$ & $35.86 \% \pm 32.92 \%$ & $57.12 \% \pm 55.35 \%$ \\
\hline & $S_{M C M}$ & $35.80 \% \pm 36.43 \%$ & $16.26 \% \pm 16.25 \%$ & $35.60 \% \pm 32.53 \%$ & $59.61 \% \pm 57.40 \%$ \\
\hline \multirow{4}{*}{$F U C$} & $S_{1}$ & $0.4593 \pm 0.0101$ & $0.4106 \pm 0.0134$ & $0.4490 \pm 0.0086$ & $0.4882 \pm 0.0119$ \\
\hline & $S_{2}$ & $0.4444 \pm 0.0000$ & $0.3970 \pm 0.0069$ & $0.4343 \pm 0.0026$ & $0.4750 \pm 0.0081$ \\
\hline & $S_{M C C}$ & $0.4541 \pm 0.0075$ & $0.4059 \pm 0.0101$ & $0.4439 \pm 0.0050$ & $0.4837 \pm 0.0086$ \\
\hline & $S_{M C M}$ & $0.4546 \pm 0.0063$ & $0.4061 \pm 0.0091$ & $0.4440 \pm 0.0047$ & $0.4836 \pm 0.0085$ \\
\hline \multirow{4}{*}{$S L U$} & $S_{1}$ & $17.9733 \pm 4.9674$ & $16.1009 \pm 4.4722$ & $17.5535 \pm 4.7830$ & $18.9526 \pm 5.1511$ \\
\hline & $S_{2}$ & $16.9132 \pm 4.1223$ & $15.2314 \pm 3.9490$ & $16.6014 \pm 4.0913$ & $18.1450 \pm 4.4514$ \\
\hline & $S_{M C C}$ & $17.5462 \pm 4.4827$ & $15.7480 \pm 4.1134$ & $17.1516 \pm 4.4176$ & $18.5518 \pm 4.8618$ \\
\hline & $S_{M C M}$ & $17.5037 \pm 4.5691$ & $15.6972 \pm 4.1480$ & $17.1442 \pm 4.4258$ & $18.5725 \pm 4.9115$ \\
\hline \multirow{4}{*}{$M L U$} & $S_{1}$ & $0.8464 \pm 0.2832$ & $0.8510 \pm 0.2759$ & $0.8790 \pm 0.2407$ & $0.9284 \pm 0.1987$ \\
\hline & $S_{2}$ & $0.4889 \pm 0.0308$ & $0.5090 \pm 0.0360$ & $0.5761 \pm 0.0303$ & $0.6313 \pm 0.0591$ \\
\hline & $S_{M C C}$ & $0.7471 \pm 0.2580$ & $0.7051 \pm 0.1859$ & $0.7656 \pm 0.1899$ & $0.8226 \pm 0.1929$ \\
\hline & $S_{M C M}$ & $0.7533 \pm 0.2322$ & $0.7071 \pm 0.1907$ & $0.7643 \pm 0.1880$ & $0.8225 \pm 0.1936$ \\
\hline \multirow{2}{*}{ Time $[\mathrm{s}]$} & $S_{M C C}$ & $4.12 \pm 2.35$ & $3.90 \pm 2.24$ & $4.10 \pm 2.34$ & $4.33 \pm 2.53$ \\
\hline & $S_{M C M}$ & $5.30 \pm 1.48$ & $4.21 \pm 2.51$ & $4.55 \pm 2.83$ & $5.07 \pm 3.37$ \\
\hline
\end{tabular}


Table 7 Network performance measure values with variation ranges, for random networks N3-2

\begin{tabular}{|c|c|c|c|c|c|}
\hline \multirow{3}{*}{$\begin{array}{c}\text { Network } \\
\text { perf. } \\
\text { measure }\end{array}$} & \multirow{3}{*}{ Method } & \multicolumn{4}{|c|}{ Baseline capacity } \\
\hline & & Fixed traffic & & Random traffic matrix & \\
\hline & & 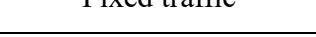 & $\min$ & avg & $\max$ \\
\hline \multirow{4}{*}{$F_{1}$} & $S_{1}$ & $13432.47 \pm 2745.74$ & $12065.21 \pm 2554.99$ & $13214.70 \pm 2713.93$ & $14472.78 \pm 3239.89$ \\
\hline & $S_{2}$ & $14436.32 \pm 3234.59$ & $13436.19 \pm 3188.43$ & $14496.68 \pm 3255.42$ & $15842.47 \pm 3624.99$ \\
\hline & $S_{M C C}$ & $13627.81 \pm 2866.45$ & $12291.44 \pm 2669.57$ & $13448.42 \pm 2836.19$ & $14762.01 \pm 3354.13$ \\
\hline & $S_{M C M}$ & $13627.41 \pm 2864.26$ & $12290.72 \pm 2677.68$ & $13447.43 \pm 2833.43$ & $14765.46 \pm 3350.44$ \\
\hline \multirow{4}{*}{$F_{2}$} & $S_{1}$ & $505675.53 \pm 107918.77$ & $367874.62 \pm 87534.55$ & $528447.90 \pm 59303.22$ & $697115.72 \pm 97679.45$ \\
\hline & $S_{2}$ & $54188.00 \pm 1792.78$ & $45979.31 \pm 1938.02$ & $64408.18 \pm 3414.14$ & $103761.14 \pm 16921.16$ \\
\hline & $S_{M C C}$ & $139316.19 \pm 30784.79$ & $99491.09 \pm 23169.63$ & $147451.20 \pm 18060.92$ & $221608.25 \pm 31964.06$ \\
\hline & $S_{M C M}$ & $139346.50 \pm 31281.87$ & $99539.71 \pm 20939.88$ & $147748.32 \pm 18982.85$ & $220217.19 \pm 33112.94$ \\
\hline \multirow{3}{*}{$R V_{1}$} & $S_{2}$ & $7.23 \% \pm 2.33 \%$ & $7.44 \% \pm 2.11 \%$ & $9.49 \% \pm 2.34 \%$ & $12.18 \% \pm 2.50 \%$ \\
\hline & $S_{M C C}$ & $1.39 \% \pm 0.66 \%$ & $1.27 \% \pm 0.57 \%$ & $1.70 \% \pm 0.61 \%$ & $2.19 \% \pm 0.57 \%$ \\
\hline & $S_{M C M}$ & $1.39 \% \pm 0.65 \%$ & $1.27 \% \pm 0.50 \%$ & $1.70 \% \pm 0.59 \%$ & $2.20 \% \pm 0.54 \%$ \\
\hline \multirow{3}{*}{$R V_{2}$} & $S_{1}$ & $832.82 \% \pm 196.30 \%$ & $498.51 \% \pm 95.62 \%$ & $742.43 \% \pm 115.26 \%$ & $987.58 \% \pm 200.37 \%$ \\
\hline & $S_{M C C}$ & $156.93 \% \pm 55.35 \%$ & $88.82 \% \pm 21.46 \%$ & $129.69 \% \pm 30.12 \%$ & $160.11 \% \pm 40.80 \%$ \\
\hline & $S_{M C M}$ & $156.94 \% \pm 55.80 \%$ & $87.72 \% \pm 22.08 \%$ & $130.38 \% \pm 31.89 \%$ & $162.27 \% \pm 42.43 \%$ \\
\hline \multirow{4}{*}{$F U C$} & $S_{1}$ & $0.6871 \pm 0.0119$ & $0.6283 \pm 0.0139$ & $0.6730 \pm 0.0135$ & $0.7260 \pm 0.0104$ \\
\hline & $S_{2}$ & $0.6667 \pm 0.0000$ & $0.6156 \pm 0.0068$ & $0.6596 \pm 0.0045$ & $0.7177 \pm 0.0104$ \\
\hline & $S_{M C C}$ & $0.6748 \pm 0.0103$ & $0.6180 \pm 0.0100$ & $0.6613 \pm 0.0100$ & $0.7142 \pm 0.0094$ \\
\hline & $S_{M C M}$ & $0.6748 \pm 0.0104$ & $0.6181 \pm 0.0105$ & $0.6613 \pm 0.0101$ & $0.7142 \pm 0.0093$ \\
\hline \multirow{4}{*}{$S L U$} & $S_{1}$ & $40.0541 \pm 1.9021$ & $36.5791 \pm 2.0405$ & $38.9164 \pm 2.1618$ & $41.9268 \pm 2.0481$ \\
\hline & $S_{2}$ & $37.4696 \pm 2.1173$ & $34.7715 \pm 1.9031$ & $37.2452 \pm 2.1259$ & $40.8238 \pm 2.6296$ \\
\hline & $S_{M C C}$ & $38.5880 \pm 2.2280$ & $35.3708 \pm 1.9735$ & $37.7421 \pm 2.3321$ & $40.8217 \pm 2.1665$ \\
\hline & $S_{M C M}$ & $38.6037 \pm 2.2217$ & $35.3614 \pm 1.9913$ & $37.7429 \pm 2.3403$ & $40.8227 \pm 2.1668$ \\
\hline \multirow{4}{*}{$M L U$} & $S_{1}$ & $1.0000 \pm 0.0000$ & $1.0000 \pm 0.0000$ & $1.0000 \pm 0.0000$ & $1.0000 \pm 0.0000$ \\
\hline & $S_{2}$ & $0.7000 \pm 0.0000$ & $0.7378 \pm 0.0532$ & $0.8157 \pm 0.0225$ & $0.9000 \pm 0.0000$ \\
\hline & $S_{M C C}$ & $0.9400 \pm 0.0680$ & $0.9175 \pm 0.0485$ & $0.9613 \pm 0.0419$ & $0.9995 \pm 0.0015$ \\
\hline & $S_{M C M}$ & $0.9400 \pm 0.0680$ & $0.9081 \pm 0.0521$ & $0.9616 \pm 0.0420$ & $1.0000 \pm 0.0000$ \\
\hline \multirow{2}{*}{ Time $[s]$} & $S_{M C C}$ & $7.72 \pm 1.20$ & $7.06 \pm 0.90$ & $7.64 \pm 0.94$ & $8.34 \pm 1.04$ \\
\hline & $S_{M C M}$ & $9.75 \pm 2.92$ & $9.15 \pm 1.73$ & $10.15 \pm 2.23$ & $11.66 \pm 2.97$ \\
\hline
\end{tabular}


Table 7 (cont.) Network performance measure values with variation ranges, for random networks N3-2

\begin{tabular}{|c|c|c|c|c|c|}
\hline \multirow{2}{*}{$\begin{array}{c}\text { Network } \\
\text { perf. } \\
\text { measure }\end{array}$} & \multirow[t]{2}{*}{ Method } & \multicolumn{4}{|c|}{$\begin{array}{l}50 \% \text { additional capacity } \\
\text { Random traffic matrix }\end{array}$} \\
\hline & & Fixe & $\min$ & avg & $\max$ \\
\hline \multirow{4}{*}{$F_{1}$} & $S_{1}$ & $13036.44 \pm 2355.63$ & $11674.57 \pm 2195.33$ & $12767.53 \pm 2313.52$ & $13877.59 \pm 2701.42$ \\
\hline & $S_{2}$ & $14164.43 \pm 3108.57$ & $12826.01 \pm 2843.38$ & $14039.90 \pm 3091.22$ & $15452.57 \pm 3519.76$ \\
\hline & $S_{M C C}$ & $13165.86 \pm 2456.07$ & $11794.07 \pm 2274.28$ & $12911.62 \pm 2425.63$ & $14061.68 \pm 2852.75$ \\
\hline & $S_{M C M}$ & $13164.65 \pm 2459.36$ & $11794.75 \pm 2272.94$ & $12910.79 \pm 2423.85$ & $14062.07 \pm 2851.10$ \\
\hline \multirow{4}{*}{$F_{2}$} & $S_{1}$ & $282342.68 \pm 160963.13$ & $201968.54 \pm 153131.42$ & $285621.35 \pm 139285.77$ & $396086.66 \pm 105496.96$ \\
\hline & $S_{2}$ & $34960.00 \pm 1156.63$ & $31881.49 \pm 1295.96$ & $34582.13 \pm 1052.33$ & $38345.84 \pm 1496.49$ \\
\hline & $S_{M C C}$ & $59741.71 \pm 23286.18$ & $49101.77 \pm 20612.28$ & $61077.51 \pm 21116.96$ & $79865.56 \pm 20654.65$ \\
\hline & $S_{M C M}$ & $60449.26 \pm 23868.43$ & $48623.76 \pm 22165.79$ & $61757.56 \pm 22519.21$ & $80280.22 \pm 23018.82$ \\
\hline \multirow{3}{*}{$R V_{1}$} & $S_{2}$ & $8.27 \% \pm 5.33 \%$ & $6.98 \% \pm 5.01 \%$ & $9.54 \% \pm 5.11 \%$ & $12.24 \% \pm 5.05 \%$ \\
\hline & $S_{M C C}$ & $0.94 \% \pm 0.89 \%$ & $0.75 \% \pm 0.79 \%$ & $1.06 \% \pm 0.91 \%$ & $1.47 \% \pm 1.05 \%$ \\
\hline & $S_{M C M}$ & $0.92 \% \pm 0.87 \%$ & $0.75 \% \pm 0.78 \%$ & $1.06 \% \pm 0.87 \%$ & $1.44 \% \pm 1.03 \%$ \\
\hline \multirow{3}{*}{$R V_{2}$} & $S_{1}$ & $708.12 \% \pm 465.35 \%$ & $499.79 \% \pm 448.95 \%$ & $721.77 \% \pm 405.40 \%$ & $982.00 \% \pm 343.91 \%$ \\
\hline & $S_{M C C}$ & $70.82 \% \pm 66.72 \%$ & $49.77 \% \pm 61.69 \%$ & $76.01 \% \pm 60.77 \%$ & $114.33 \% \pm 54.55 \%$ \\
\hline & $S_{M C M}$ & $72.89 \% \pm 68.67 \%$ & $48.71 \% \pm 67.34 \%$ & $77.92 \% \pm 64.85 \%$ & $113.66 \% \pm 63.02 \%$ \\
\hline \multirow{4}{*}{$F U C$} & $S_{1}$ & $0.4732 \pm 0.0126$ & $0.4309 \pm 0.0139$ & $0.4624 \pm 0.0135$ & $0.5007 \pm 0.0118$ \\
\hline & $S_{2}$ & $0.4444 \pm 0.0000$ & $0.4036 \pm 0.0050$ & $0.4351 \pm 0.0021$ & $0.4732 \pm 0.0053$ \\
\hline & $S_{M C C}$ & $0.4595 \pm 0.0118$ & $0.4178 \pm 0.0091$ & $0.4495 \pm 0.0106$ & $0.4870 \pm 0.0086$ \\
\hline & $S_{M C M}$ & $0.4594 \pm 0.0118$ & $0.4179 \pm 0.0092$ & $0.4494 \pm 0.0109$ & $0.4867 \pm 0.0094$ \\
\hline \multirow{4}{*}{$S L U$} & $S_{1}$ & $28.8153 \pm 1.9658$ & $26.4379 \pm 2.2847$ & $28.0649 \pm 2.1838$ & $30.5958 \pm 2.0203$ \\
\hline & $S_{2}$ & $25.0790 \pm 1.5582$ & $22.9592 \pm 1.3793$ & $24.7352 \pm 1.5634$ & $27.1039 \pm 1.5360$ \\
\hline & $S_{M C C}$ & $27.4619 \pm 2.1604$ & $25.0250 \pm 2.1415$ & $26.7341 \pm 2.0879$ & $29.1777 \pm 1.9573$ \\
\hline & $S_{M C M}$ & $27.5081 \pm 2.1771$ & $25.0117 \pm 2.2112$ & $26.7414 \pm 2.1083$ & $29.1712 \pm 1.9464$ \\
\hline \multirow{4}{*}{$M L U$} & $S_{1}$ & $1.0000 \pm 0.0000$ & $1.0000 \pm 0.0000$ & $1.0000 \pm 0.0000$ & $1.0000 \pm 0.0000$ \\
\hline & $S_{2}$ & $0.5000 \pm 0.0000$ & $0.5467 \pm 0.0563$ & $0.5950 \pm 0.0064$ & $0.6000 \pm 0.0000$ \\
\hline & $S_{M C C}$ & $0.8578 \pm 0.0657$ & $0.8212 \pm 0.0731$ & $0.8633 \pm 0.0586$ & $0.9013 \pm 0.0350$ \\
\hline & $S_{M C M}$ & $0.8578 \pm 0.0657$ & $0.7917 \pm 0.0927$ & $0.8609 \pm 0.0569$ & $0.8986 \pm 0.0320$ \\
\hline \multirow{2}{*}{ Time $[\mathrm{s}]$} & $S_{M C C}$ & $6.10 \pm 0.88$ & $5.69 \pm 0.63$ & $6.01 \pm 0.65$ & $6.53 \pm 0.77$ \\
\hline & $S_{M C M}$ & $7.75 \pm 2.81$ & $6.64 \pm 1.93$ & $7.51 \pm 2.07$ & $8.47 \pm 2.43$ \\
\hline
\end{tabular}


Table 8 Network performance measure values with variation ranges, for random networks N3-4

\begin{tabular}{|c|c|c|c|c|c|}
\hline $\begin{array}{l}\text { Network } \\
\text { perf. } \\
\text { measure }\end{array}$ & Method & Fixed traffic & Baseline & $\begin{array}{l}\text { capacity } \\
\text { Random traffic matrix } \\
\text { avg }\end{array}$ & $\max$ \\
\hline \multirow{4}{*}{$F_{1}$} & $S_{1}$ & $75528.34 \pm 3666.64$ & $70818.03 \pm 3894.02$ & $74427.07 \pm 3572.53$ & $77379.17 \pm 3113.92$ \\
\hline & $S_{2}$ & $82466.26 \pm 3717.76$ & $79240.25 \pm 3678.59$ & $82991.42 \pm 3674.71$ & $86417.03 \pm 3403.72$ \\
\hline & $S_{M C C}$ & $76652.50 \pm 3606.71$ & $71984.76 \pm 3843.55$ & $75719.15 \pm 3509.45$ & $78812.30 \pm 3087.91$ \\
\hline & $S_{M C M}$ & $76656.74 \pm 3568.04$ & $72009.20 \pm 3802.63$ & $75736.65 \pm 3491.48$ & $78793.59 \pm 3067.92$ \\
\hline \multirow{4}{*}{$F_{2}$} & $S_{1}$ & $3597953.87 \pm 522542.37$ & $2737396.67 \pm 317666.43$ & $3319054.65 \pm 359652.15$ & $3729376.96 \pm 412802.13$ \\
\hline & $S_{2}$ & $335668.00 \pm 23003.53$ & $277049.67 \pm 21108.16$ & $325991.93 \pm 21339.77$ & $369563.44 \pm 24404.60$ \\
\hline & $S_{M C C}$ & $865480.25 \pm 117267.99$ & $621739.72 \pm 62999.61$ & $793227.55 \pm 83639.06$ & $905634.66 \pm 93635.06$ \\
\hline & $S_{M C M}$ & $863968.66 \pm 108085.70$ & $615701.30 \pm 70762.97$ & $785835.34 \pm 74929.49$ & $905728.23 \pm 97371.59$ \\
\hline \multirow{3}{*}{$R V_{1}$} & $S_{2}$ & $9.21 \% \pm 2.17 \%$ & $10.36 \% \pm 2.70 \%$ & $11.54 \% \pm 2.75 \%$ & $12.60 \% \pm 2.81 \%$ \\
\hline & $S_{M C C}$ & $1.49 \% \pm 0.34 \%$ & $1.52 \% \pm 0.44 \%$ & $1.74 \% \pm 0.40 \%$ & $1.98 \% \pm 0.42 \%$ \\
\hline & $S_{M C M}$ & $1.50 \% \pm 0.42 \%$ & $1.56 \% \pm 0.44 \%$ & $1.76 \% \pm 0.41 \%$ & $1.99 \% \pm 0.45 \%$ \\
\hline \multirow{3}{*}{$R V_{2}$} & $S_{1}$ & $973.80 \% \pm 167.80 \%$ & $841.15 \% \pm 139.60 \%$ & $921.87 \% \pm 143.31 \%$ & $1026.12 \% \pm 190.74 \%$ \\
\hline & $S_{M C C}$ & $158.13 \% \pm 35.48 \%$ & $121.27 \% \pm 14.80 \%$ & $143.31 \% \pm 24.76 \%$ & $163.26 \% \pm 33.01 \%$ \\
\hline & $S_{M C M}$ & $157.43 \% \pm 28.23 \%$ & $114.48 \% \pm 14.13 \%$ & $141.00 \% \pm 22.75 \%$ & $164.87 \% \pm 27.57 \%$ \\
\hline \multirow{4}{*}{$F U C$} & $S_{1}$ & $0.6920 \pm 0.0099$ & $0.6505 \pm 0.0083$ & $0.6799 \pm 0.0103$ & $0.7020 \pm 0.0106$ \\
\hline & $S_{2}$ & $0.6667 \pm 0.0000$ & $0.6252 \pm 0.0036$ & $0.6560 \pm 0.0014$ & $0.6789 \pm 0.0035$ \\
\hline & $S_{M C C}$ & $0.6776 \pm 0.0033$ & $0.6366 \pm 0.0042$ & $0.6659 \pm 0.0046$ & $0.6878 \pm 0.0058$ \\
\hline & $S_{M C M}$ & $0.6775 \pm 0.0031$ & $0.6365 \pm 0.0043$ & $0.6658 \pm 0.0046$ & $0.6880 \pm 0.0061$ \\
\hline \multirow{4}{*}{$S L U$} & $S_{1}$ & $85.5578 \pm 9.2453$ & $80.5862 \pm 8.9263$ & $84.2721 \pm 9.4491$ & $86.9279 \pm 9.4606$ \\
\hline & $S_{2}$ & $81.7768 \pm 8.1332$ & $76.7942 \pm 7.4735$ & $80.3500 \pm 7.9643$ & $83.0299 \pm 8.1954$ \\
\hline & $S_{M C C}$ & $83.6162 \pm 8.9559$ & $78.6404 \pm 8.2784$ & $82.2108 \pm 8.9423$ & $84.5953 \pm 8.7675$ \\
\hline & $S_{M C M}$ & $83.6151 \pm 8.9019$ & $78.6233 \pm 8.2677$ & $82.1982 \pm 8.9562$ & $84.6888 \pm 8.7708$ \\
\hline \multirow{4}{*}{$M L U$} & $S_{1}$ & $1.0000 \pm 0.0000$ & $1.0000 \pm 0.0000$ & $1.0000 \pm 0.0000$ & $1.0000 \pm 0.0000$ \\
\hline & $S_{2}$ & $0.7000 \pm 0.0000$ & $0.7008 \pm 0.0023$ & $0.7654 \pm 0.0213$ & $0.8202 \pm 0.0320$ \\
\hline & $S_{M C C}$ & $0.9050 \pm 0.0140$ & $0.9000 \pm 0.0000$ & $0.9032 \pm 0.0054$ & $0.9306 \pm 0.0520$ \\
\hline & $S_{M C M}$ & $0.9000 \pm 0.0000$ & $0.9000 \pm 0.0000$ & $0.9009 \pm 0.0025$ & $0.9094 \pm 0.0244$ \\
\hline \multirow{2}{*}{ Time $[\mathrm{s}]$} & $S_{M C C}$ & $97.38 \pm 10.36$ & $87.09 \pm 5.34$ & $92.20 \pm 5.64$ & $96.94 \pm 7.10$ \\
\hline & $S_{M C M}$ & $132.60 \pm 38.73$ & $98.74 \pm 16.22$ & $119.01 \pm 20.74$ & $146.43 \pm 31.22$ \\
\hline
\end{tabular}


Table 8 (cont.) Network performance measure values with variation ranges, for random networks N3-4

\begin{tabular}{|c|c|c|c|c|c|}
\hline \multirow{2}{*}{$\begin{array}{c}\text { Network } \\
\text { perf. } \\
\text { measure }\end{array}$} & \multirow[b]{2}{*}{ Method } & \multicolumn{4}{|c|}{$50 \%$ additional capacity } \\
\hline & & Fixed traffic & $\min$ & $\begin{array}{c}\text { Random traffic matrix } \\
\text { avg }\end{array}$ & $\max$ \\
\hline \multirow{4}{*}{$F_{1}$} & $S_{1}$ & $74431.25 \pm 3797.46$ & $69776.41 \pm 3954.76$ & $73251.24 \pm 3752.07$ & $76028.31 \pm 3446.19$ \\
\hline & $S_{2}$ & $79596.30 \pm 3311.24$ & $74577.81 \pm 3874.77$ & $79016.75 \pm 3419.07$ & $82540.96 \pm 3225.18$ \\
\hline & $S_{M C C}$ & $75018.41 \pm 3756.90$ & $70307.07 \pm 3933.84$ & $73872.70 \pm 3701.21$ & $76723.27 \pm 3375.08$ \\
\hline & $S_{M C M}$ & $74995.24 \pm 3796.19$ & $70319.18 \pm 3926.18$ & $73855.64 \pm 3718.57$ & $76709.64 \pm 3362.82$ \\
\hline \multirow{4}{*}{$F_{2}$} & $S_{1}$ & $937868.75 \pm 395407.93$ & $736965.12 \pm 286168.26$ & $955039.63 \pm 317084.83$ & $1193586.36 \pm 323364.65$ \\
\hline & $S_{2}$ & $216560.00 \pm 14840.99$ & $202987.09 \pm 13954.96$ & $212912.09 \pm 14289.26$ & $220396.64 \pm 15360.76$ \\
\hline & $S_{M C C}$ & $294882.27 \pm 44316.35$ & $262100.97 \pm 26828.77$ & $289610.74 \pm 38545.81$ & $315037.63 \pm 46180.37$ \\
\hline & $S_{M C M}$ & $298653.53 \pm 48461.38$ & $263729.99 \pm 31236.58$ & $291526.83 \pm 39204.28$ & $317231.61 \pm 44275.68$ \\
\hline \multirow{3}{*}{$R V_{1}$} & $S_{2}$ & $6.97 \% \pm 1.91 \%$ & $6.49 \% \pm 1.63 \%$ & $7.89 \% \pm 2.11 \%$ & $9.34 \% \pm 2.69 \%$ \\
\hline & $S_{M C C}$ & $0.79 \% \pm 0.21 \%$ & $0.68 \% \pm 0.18 \%$ & $0.85 \% \pm 0.23 \%$ & $1.00 \% \pm 0.30 \%$ \\
\hline & $S_{M C M}$ & $0.76 \% \pm 0.17 \%$ & $0.65 \% \pm 0.18 \%$ & $0.83 \% \pm 0.22 \%$ & $0.98 \% \pm 0.25 \%$ \\
\hline \multirow{3}{*}{$R V_{2}$} & $S_{1}$ & $331.80 \% \pm 171.46 \%$ & $250.47 \% \pm 124.27 \%$ & $347.48 \% \pm 140.51 \%$ & $454.56 \% \pm 143.98 \%$ \\
\hline & $S_{M C C}$ & $36.03 \% \pm 15.27 \%$ & $26.73 \% \pm 9.01 \%$ & $35.88 \% \pm 13.33 \%$ & $46.17 \% \pm 17.16 \%$ \\
\hline & $S_{M C M}$ & $37.77 \% \pm 17.64 \%$ & $27.66 \% \pm 10.34 \%$ & $36.79 \% \pm 13.89 \%$ & $46.81 \% \pm 15.88 \%$ \\
\hline \multirow{4}{*}{$F U C$} & $S_{1}$ & $0.4672 \pm 0.0081$ & $0.4386 \pm 0.0071$ & $0.4592 \pm 0.0085$ & $0.4748 \pm 0.0082$ \\
\hline & $S_{2}$ & $0.4444 \pm 0.0000$ & $0.4165 \pm 0.0025$ & $0.4368 \pm 0.0010$ & $0.4520 \pm 0.0023$ \\
\hline & $S_{M C C}$ & $0.4597 \pm 0.0067$ & $0.4326 \pm 0.0052$ & $0.4520 \pm 0.0069$ & $0.4671 \pm 0.0058$ \\
\hline & $S_{M C M}$ & $0.4599 \pm 0.0069$ & $0.4324 \pm 0.0048$ & $0.4522 \pm 0.0070$ & $0.4674 \pm 0.0064$ \\
\hline \multirow{4}{*}{$S L U$} & $S_{1}$ & $58.3914 \pm 6.8361$ & $54.8094 \pm 6.2456$ & $57.4199 \pm 6.7917$ & $59.3277 \pm 6.8259$ \\
\hline & $S_{2}$ & $54.5301 \pm 5.6589$ & $51.0505 \pm 5.2117$ & $53.5935 \pm 5.5920$ & $55.2742 \pm 5.6446$ \\
\hline & $S_{M C C}$ & $57.1587 \pm 6.4050$ & $53.7229 \pm 5.9307$ & $56.1948 \pm 6.4298$ & $58.0616 \pm 6.3418$ \\
\hline & $S_{M C M}$ & $57.1803 \pm 6.4128$ & $53.6978 \pm 5.9092$ & $56.2214 \pm 6.4377$ & $58.1055 \pm 6.3777$ \\
\hline \multirow{4}{*}{$M L U$} & $S_{1}$ & $1.0000 \pm 0.0000$ & $1.0000 \pm 0.0000$ & $1.0000 \pm 0.0000$ & $1.0000 \pm 0.0000$ \\
\hline & $S_{2}$ & $0.5000 \pm 0.0000$ & $0.5000 \pm 0.0000$ & $0.5251 \pm 0.0218$ & $0.5769 \pm 0.0375$ \\
\hline & $S_{M C C}$ & $0.7939 \pm 0.0648$ & $0.7800 \pm 0.0555$ & $0.8026 \pm 0.0477$ & $0.8373 \pm 0.0636$ \\
\hline & $S_{M C M}$ & $0.8011 \pm 0.0737$ & $0.7800 \pm 0.0555$ & $0.8034 \pm 0.0416$ & $0.8374 \pm 0.0638$ \\
\hline \multirow{2}{*}{ Time [s] } & $S_{M C C}$ & $67.13 \pm 4.97$ & $64.39 \pm 3.32$ & $67.86 \pm 3.64$ & $71.42 \pm 4.19$ \\
\hline & $S_{M C M}$ & $96.61 \pm 8.80$ & $88.65 \pm 3.75$ & $97.86 \pm 4.09$ & $107.55 \pm 5.30$ \\
\hline
\end{tabular}




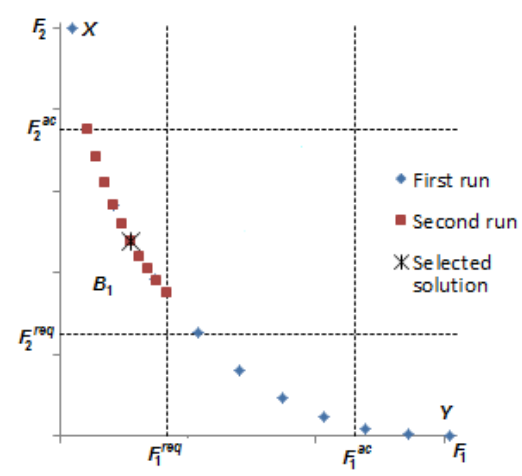

(a) Solutions obtained with the MCC algorithm in the situation of baseline link capacity (increased by 20\%)

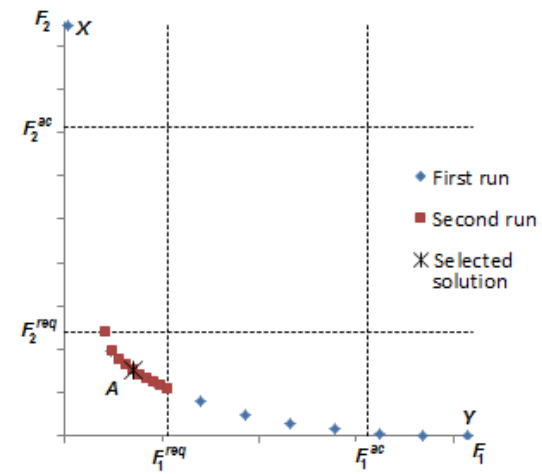

(c) Solutions obtained with the MCC algorithm in the situation of $50 \%$ additional link capacity

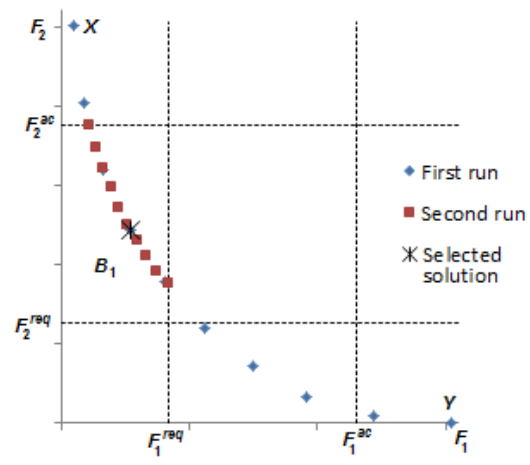

(b) Solutions obtained with the MCM algorithm in the situation of baseline link capacity (increased by 20\%)

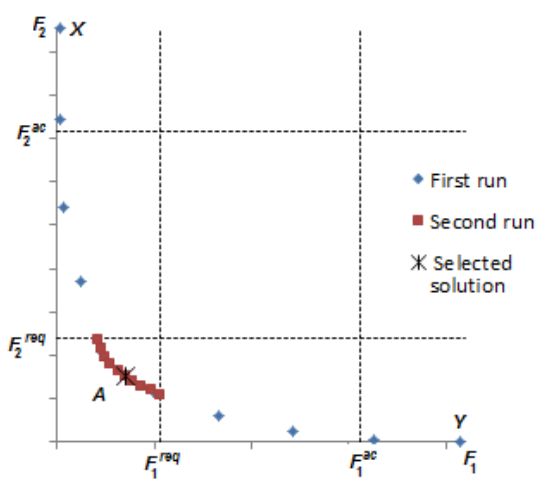

(d) Solutions obtained with the MCM algorithm in the situation of $50 \%$ additional link capacity

Figure 14 Non-dominated solutions obtained for fixed traffic in network $\mathrm{N} 1-1 \mathrm{~b}$

of the algorithm (see figures 14a and 14c-14d).

The final solutions obtained by the two methods are in general different but normally very close to each other and leading to very similar network performances. This similarity of the final solutions was expectable since both are exact methods for generating non-dominated solutions, the degree of 'exploration' of the decision space was defined with the same parametrization in the corresponding sets of experiments and the method for the final solution selection was the same, consisting of an automated procedure based on a reference point type approach.

The final solutions obtained with the $M C C$ and $M C M$ algorithms are both guaranteed to be nondominated solutions. There is not a method that is clearly better than the other in terms of running times, 
although we might mention the fact that the $M C M$ algorithm tends to take more time to run in the great majority of the experiments (the exceptions being for network N1-3) but the differences in average running times are not significant.

As for the running time of the algorithms, it clearly increases when the number of nodes and links increases. In the situations where a $50 \%$ additional capacity in the links is considered, the running time is lower than when the baseline capacity of the links is considered. As the capacity of the links increases, it is easier to "accommodate" the offered traffic, and it is faster to obtain an adequate solution.

\section{Conclusions}

In this paper, we presented a multiobjective routing model for MPLS networks with different service types considering a network-wide optimization approach based on a network flow modeling approach. This model may be relevant for telecommunication operators. In many currently implemented systems, the routing is optimized using a flow-oriented based approach, which lacks a global perspective in terms of global network performance/cost metrics. The routing problem is formulated as a multiobjective mixed-integer program, where the considered objectives were the minimization of the bandwidth transport cost and the minimization of the total load cost in the network links, with a constraint on the maximal splitting of traffic trunks. A variant of the original problem was proposed and two different exact methods were developed for solving the formulated problem, the $M C C$ algorithm and the $M C M$ algorithm, based on the classical constraint method and on a modified constraint method, respectively.

A very extensive experimental study was presented, considering reference test networks and MPLS networks randomly generated with the gt-itm software. Results on relevant network performance measures (some related to the link utilization, and also the relative variation of the obtained solutions when compared to the optimal solutions obtained when each of the objective functions is individually optimized) were presented.

The obtained results show that the objective functions $F_{1}$ and $F_{2}$ are indeed conflicting, which confirms the potential advantages of using these two objectives in this routing model, rather than solving a single objective problem. The fact that we are using two different and conflicting objectives provides the decision maker with the tools to understand the implications of these two aspects: on the one hand, the minimization of the bandwidth transport cost and on the other hand, the minimization of the load cost in the network links. If only the first objective was taken into account, there would be the tendency to use shortest paths, which would cause some links to be over-utilized while others would remain underutilized. By including the second objective function, we aim at an adequate load distribution in the network, which would lead to a more balanced use of the network links. This way, the trade-offs among these distinct performance metrics can be analyzed in a consistent manner.

The numerical values of the network performance measures calculated for the obtained solutions are relevant, because they give a quantitative measure of the trade-offs between the two objective functions, as well as the level attained by significant performance metrics. In particular, the $M L U$ is a relevant measurement in traffic engineering terms and it shows that the minimization of $F_{2}$ leads indeed to a 
better load distribution in the network, at the cost of an increase in the bandwidth transport cost.

We can conclude that none of the algorithms $M C C$ or $M C M$ is clearly better than the other since they not only lead to similar solutions in terms of network performance as could be expected, but also the running times are similar, with a slight advantage to $M C C$. The final solutions are similar as both resolution methods are exact methods for generating non-dominated solutions, and also because the same parametrization was used to explore the decision space and the final solution was selected with the same method.

Note that both methods could be easily adapted to an interactive procedure for selecting a final nondominated solution, as in a non-automated off-line application environment. In this case, the decision maker would explore the Pareto front at will and could favour different solutions representing different trade-offs between routing cost and total load cost. Also note that the dimension of the range of solutions that can be explored can be widened straightforwardly by increasing the parameter $\Delta$ in both algorithms.

In this context, the only advantage of $M C M$ over $M C C$ stems from the fact that it tends to generate more evenly spaced solutions in the Pareto frontier.

Future developments of this work will involve the adaptation of the model to the routing of incremental type traffic and the formulation of a multiobjective model of similar nature for networks with two service classes in terms of bandwidth assignment priority, namely QoS and BE traffic. Finally, different specifications of the objective functions could be considered for different network application environments.

\section{Acknowledgments}

This work was financially supported by programme COMPETE of the EC Community Support Framework III and cosponsored by the EC fund FEDER and national funds (Portuguese Foundation for Science and Technology under projects PTDC/EEA-TEL/101884/2008, PEst- OE/EEI/UI0308/2014 and PEst-OE/MAT/ UI0152). The authors would like to thank the anonymous referees for their comments, which have helped to improve the quality of the paper.

\section{References}

[1] Aarts, E. \& Lenstra, J. K. (editors). Local Search in Combinatorial Optimization. John Wiley \& Sons, 1997.

[2] Agdeppa, R. P., Yamashita, N. \& Fukushima, M. The traffic equilibrium problem with nonadditive costs and its monotone mixed complementarity problem formulation. Transportation Research Part B - Methodological, 41(8):862-874, 2007.

[3] Avallone, S., Manetti, V., Mariano, M. \& Romano, S. P. A splitting infrastructure for load balancing and security in an MPLS network. Proceedings of the 3rd International Conference on Testbeds and Research Infrastructure for the Development of Networks and Communities (TridentCom 2007), Lake Buena Vista (FL), USA, May 21-23, 2007. 
[4] Bekhor, S., Toledo, T. \& Toledo, J. N. Effects of choice set size and route choice models on pathbased traffic assignment. Transportmetrica, 4(2):117-133, 2008.

[5] Bertsimas, D. \& Tsitsiklis, J. Simulated annealing. Statistical Science, 8(1):10-15, 1993.

[6] Bovy, P. H. L. On modelling route choice sets in transportation networks: A synthesis. Transport Reviews: A Transnational Transdisciplinary Journal, 29(1):43-68, 2009.

[7] Brands, T. \& van Eck, G. Multimodal network design and assessment - Proposal for a dynamic multi-objective approach. 11th TRAIL Congress, The Netherlands Research School on Transport, Infrastructure and Logistics, Nov. 2010.

[8] Branke, J., Deb, K., Miettinen, K. \& Słowiński, R. (editors). Multiobjective Optimization Interactive and Evolutionary Approaches. Lecture Notes in Computer Science, volume 5252, Springer, 2008.

[9] Chen, A., Zhou, Z., Chootinan, P., Ryu, S., Yang, C. \& Wong, S. C. Transport network design problem under uncertainty: A review and new developments. Transport Reviews, 31(6):743-768, 2011.

[10] Clímaco, J. C. N., Craveirinha, J. M. F. \& Pascoal, M. M. B. An automated reference point-like approach for multicriteria shortest path problems. Journal of Systems Science and Systems Engineering, 15(3):314-329. 2006.

[11] Clímaco, J. C. N., Craveirinha, J. M. F. \& Pascoal, M. M. B. Multicriteria routing models in telecommunication networks - Overview and a case study. In Shi, Y., Olson, D. L. \& Stam, A. (editors), Advances in Multiple Criteria Decision Making and Human Systems Management: Knowledge and Wisdom, pages 17-46, IOS Press, 2007.

[12] Clímaco, J. \& Pascoal, M. Finding nondominated shortest pairs of disjoint simple paths. Computers \& Operations Research, 36(11):2892-2898, 2009.

[13] Cohon, J. L. Multiobjective Programming and Planning. Mathematics in Science and Engineering, Academic Press, 1978.

[14] Craveirinha, J., Gomes, T., Pascoal, M. \& Clímaco, J. A stochastic bicriteria approach for restorable QoS routing in MPLS. Proceedings of the 2011 International Conference on Telecommunication Systems - Modeling and Analysis (ICTSM2011), pages 1-15, Prague, Czech Republic, May 26-28, 2011.

[15] Craveirinha, J., Clímaco, J., Martins, L., da Silva, C. G. \& Ferreira, N. A bi-criteria minimum spanning tree routing model for MPLS/Overlay networks. Telecommunication Systems, 52(1):203$215,2013$.

[16] Craveirinha, J. M. F., Clímaco, J. C. N., Pascoal, M. M. B. \& Martins, L. M. R. A. Traffic splitting in MPLS networks - A hierarchical multicriteria approach. Journal of Telecommunications and Information Technology, (4):3-10, 2007.

[17] Craveirinha, J., Girão-Silva, R. \& Clímaco, J. A meta-model for multiobjective routing in MPLS networks. Central European Journal of Operations Research, 16(1):79-105, 2008. 
[18] Dana, A., Zadeh, A. K., Kalantari, M. E. \& Badie, K. A traffic splitting restoration scheme for MPLS network using case-based reasoning. Proceedings of the 9th Asia Pacific Conference on Communications (APCC 2003), volume 2, pages 763-766, Sep. 21-24, 2003.

[19] Dana, A., Khademzadeh, A., Kalantari, M. E. \& Badie, K. Fault recovery in MPLS network using case-based reasoning. Modares Technical and Engineering, 16:127-138, 2004.

[20] Deb, K. Multi-Objective Optimization using Evolutionary Algorithms. John Wiley \& Sons, 2001.

[21] Dial, R. B. Bicriterion traffic assignment: Basic theory and elementary algorithms. Transportation Science, 30(2):93-111, 1996.

[22] Dial, R. B. Bicriterion traffic assignment: Efficient algorithms plus examples. Transportation Research Part B - Methodological, 31(5):357-379, 1997.

[23] Dixit, A., Prakash, P. \& Kompella, R. R. On the efficacy of fine-grained traffic splitting protocols in data center networks. Proceedings of SIGCOMM11, pages 430-431, Toronto (Ontario), Canada, Aug. 15-19, 2011.

[24] Doar, M. \& Leslie, I. M. How bad is naive multicast routing? Proceedings of INFOCOM, volume 1, pages 82-89, San Francisco (CA), USA, 1993.

[25] Ehrgott, M. \& Gandibleux, X. A survey and annotated bibliography of multiobjective combinatorial optimization. OR Spektrum, 22(4):425-460, 2000.

[26] Elwalid, A., Jin, C., Low, S. \& Widjaja, I. MATE: MPLS Adaptive Traffic Engineering. In Sengupta, B., Bauer, F. \& Cavendish, D. (editors), Proceedings of the 20th Annual Joint Conference of the IEEE Computer and Communications Societies (INFOCOM 2001), volume 3, pages 1300 1309, Anchorage (AK), USA, IEEE Computer and Communications Societies, 2001.

[27] Erbas, S. C. \& Erbas, C. A multiobjective off-line routing model for MPLS networks. In Charzinski, J., Lehnert, R. \& Tran-Gia, P. (editors), Proceedings of the 18th International Teletraffic Congress (ITC-18), pages 471-480, Berlin, Germany, Elsevier, Amsterdam, 2003.

[28] Ferng, H.-W. \& Peng, C.-C. Traffic splitting in a network: Split traffic models and applications. Computer Communications, 27(12):1152-1165, 2004.

[29] Fortz, B. \& Thorup, M. Internet traffic engineering by optimizing OSPF weights. In Sidi, M., Katzela, I. \& Shavitt, Y. (editors), Proceedings of the 19th Annual Joint Conference of the IEEE Computer and Communications Societies (INFOCOM 2000), volume 2, pages 519-528, Tel Aviv, Israel, Mar. 26-30, IEEE Computer and Communications Societies, 2000.

[30] Fortz, B. \& Thorup, M. Optimizing OSPF/IS-IS weights in a changing world. IEEE Journal on Selected Areas in Communications, 20(4):756-767, 2002.

[31] Gandibleux, X., Sevaux, M., Sörensen, K. \& T'Kindt, V. (editors). Metaheuristics for Multiobjective Optimisation. Lecture Notes in Economics and Mathematical Systems, volume 535, Springer, 2004.

[32] Gendreau, M. \& Potvin, J.-Y. Tabu search. In Gendreau, M. \& Potvin, J.-Y. (editors), Handbook of Metaheuristics, International Series in Operations Research \& Management Science, volume 146, pages 41-59, Springer, 2010. 
[33] Ghosh, A. \& Dehuri, S. Evolutionary algorithms for multi-criterion optimization: A survey. International Journal of Computing \& Information Sciences, 2(1):38-57, 2004.

[34] Girão-Silva, R., Craveirinha, J. \& Clímaco, J. Hierarchical multiobjective routing in Multiprotocol Label Switching networks with two service classes - A heuristic solution. International Transactions in Operational Research, 16(3):275-305, 2009.

[35] Girão-Silva, R., Craveirinha, J., Clímaco, J. \& Captivo, M. E. Multiobjective Routing in Multiservice MPLS Networks with Traffic Splitting - Report on a Network Flow Approach. Research Report 2/2013, INESC-Coimbra, 2013.

[36] Gomes, T., Martins, L. \& Craveirinha, J. An algorithm for calculating $k$ shortest paths with a maximum number of arcs. Investigação Operacional, 21:235-244, 2001.

[37] Guihaire, V. \& Hao, J.-K. Transit network design and scheduling: a global review. Transportation Research Part A: Policy and Practice, 42(10):1251-1273, 2008.

[38] He, J. \& Rexford, J. Towards Internet-wide multipath routing. IEEE Network, 22(2):16-21, 2008.

[39] Huang, H.-J. \& Li, Z.-C. A multiclass, multicriteria logit-based traffic equilibrium assignment model under ATIS. European Journal of Operational Research, 176(3):1464-1477, 2007.

[40] Knowles, J., Oates, M. \& Corne, D. Advanced multi-objective evolutionary algorithms applied to two problems in telecommunications. BT Technology Journal, 18(4):51-65, 2000.

[41] Krishnadas, C. S. \& Roy, R. Quality of Experience (QoE) assurance by a multipath balanced traffic splitting algorithm in MPLS networks. Annales UMCS Informatica AI, 9(1):165-177, 2009.

[42] Lee, G. M. \& Choi, J. S. A survey of multipath routing for traffic engineering. [Online], 2002.

[43] Lee, Y., Seok, Y., Choi, Y. \& Kim, C. A constrained multipath traffic engineering scheme for MPLS networks. Proceedings of the IEEE International Conference on Communications (ICC 2002), New York, USA, Apr.28-May2, 2002.

[44] Lee, K., Toguyeni, A., Noce, A. \& Rahmani, A. Comparison of multipath algorithms for load balancing in a MPLS network. In Kim, C. (editor), Proceedings of the International Conference on Information Networking, Convergence in Broadband and Mobile Networking (ICOIN2005), Lecture Notes in Computer Science, volume 3391, pages 463-470, Jeju Island, Korea, Jan.31-Feb.2, Springer, 2005.

[45] Lee, K., Toguyeni, A. \& Rahmani, A. Hybrid multipath routing algorithms for load balancing in MPLS based IP network. Proceedings of the 20th International Conference on Advanced Information Networking and Applications (AINA 2006), Apr.18-20, 2006.

[46] Liu, Y., Bunker, J. \& Ferreira, L. Transit users' route-choice modelling in transit assignment: A review. Transport Reviews: A Transnational Transdisciplinary Journal, 30(6):753-769, 2010.

[47] Lo, H. K. \& Chen, A. Reformulating the traffic equilibrium problem via a smooth gap function. Mathematical and Computer Modelling, 31(2-3):179-195, 2000.

[48] Lo, H. K. \& Chen, A. Traffic equilibrium problems with route-specific costs: formulation and algorithms. Transportation Research Part B - Methodological, 34(6):493-513, 2000. 
[49] Lu, C.-C., Mahmassani, H. S. \& Zhou, X. A bi-criterion dynamic user equilibrium traffic assignment model and solution algorithm for evaluating dynamic road pricing strategies. Transportation Research Part C, 16:371-389, 2008.

[50] Marcotte, P. \& Patriksson, M. Traffic equilibrium. In Barnhart, C. \& Laporte, G. (editors), Transportation, Handbooks in Operations Research and Management Science, volume 14, pages 623713, North-Holland, Amsterdam, 2007.

[51] Mavrotas, G. Effective implementation of the $\varepsilon$-constraint method in Multi-Objective Mathematical Programming problems. Applied Mathematics and Computation, 213(2):455-465, 2009.

[52] Medhi, D. \& Tipper, D. Some approaches to solving a multi-hour broadband network capacity design problem with single-path routing. Telecommunication Systems, 13(2):269-291, 2000.

[53] Messac, A., Ismail-Yahaya, A. \& Mattson, C. A. The normalized normal constraint method for generating the Pareto frontier. Structural and Multidisciplinary Optimization, 25(2):86-98, 2003.

[54] Mitra, D. \& Ramakrishnan, K. G. Techniques for traffic engineering of multiservice, multipriority networks. Bell Labs Technical Journal, 6(1):139-151, 2001.

[55] Murugesan, G., Natarajan, A. M. \& Venkatesh, C. Enhanced variable splitting ratio algorithm for effective load balancing in MPLS networks. Journal of Computer Science, 4(3):232-238, 2008.

[56] Nagurney, A., Dong, J. \& Mokhtarian, P. L. Traffic network equilibrium and the environment: A multicriteria decision- making perspective. In Kontoghiorghes, E., Rustem, B. \& Siokos, S. (editors), Computational Methods in Decision-Making, Economics and Finance, pages 501-523, Kluwer, 2002.

[57] Nelakuditi, S. \& Zhang, Z.-L. On selection of paths for multipath routing. In Wolf, L., Hutchison, D. \& Steinmetz, R. (editors), Proceedings of IWQoS 2001, Lecture Notes in Computer Science, volume 2092, pages 170-184, Karlsruhe, Germany, Springer, 2001.

[58] Patriksson, M. The Traffic Assignment Problem - Models and Methods. Topics in Transportation, VSP, 1994.

[59] Pióro, M., Szentesi, Á., Harmatos, J., Jüttner, A., Gajowniczek, P. \& Kozdrowski, S. On open shortest path first related network optimization problems. Performance Evaluation, 48:201-223, 2002.

[60] Prashker, J. N. \& Bekhor, S. A review on route choice models used in the stochastic user equilibrium problem. Transport Reviews, 24(4):437-463, 2004.

[61] Prato, C. G. Route choice modeling: past, present and future research directions. Journal of Choice Modelling, 2(1):65-100, 2009.

[62] Raith, A., Wang, J. Y. T., Ehrgott, M. \& Mitchell, S. A. Solving multi-objective traffic assignment. In ORP3 Meeting, Cádiz, Spain, Sep.13-17, 2011.

[63] Ran, B. \& Boyce, D. Modeling Dynamic Transportation Networks - An Intelligent Transportation System Oriented Approach. Lecture Notes in Economics and Mathematical Systems, volume 417, Springer, 2nd ed., 1996.

[64] Sheffi, Y. Urban Transportation Networks: Equilibrium Analysis with Mathematical Programming Methods. Prentice-Hall, Inc., 1985. 
[65] Singh, R. K., Chaudhari, N. S. \& Saxena, K. Load balancing in IP/MPLS networks: A survey. Communications and Network, 4:151-156, 2012.

[66] Song, J., Kim, S. \& Lee, M. Dynamic load distribution in MPLS networks. In Kahng, H.-K. (editor), Proceedings of the International Conference on Information Networking, Convergence in Broadband and Mobile Networking (ICOIN2003), Lecture Notes in Computer Science, volume 2662, pages 989-999, Jeju Island, Korea, Feb.12-14, Springer, 2003.

[67] Srivastava, S., Krithikaivasan, B., Medhi, D. \& Pióro, M. Traffic engineering in the presence of tunneling and diversity constraints: Formulation and Lagrangean decomposition approach. In Charzinski, J., Lehnert, R. \& Tran-Gia, P. (editors), Proceedings of the 18th International Teletraffic Congress (ITC-18), pages 461-470, Berlin, Germany, Elsevier, Amsterdam, 2003.

[68] Srivastava, S., Agrawal, G., Pióro, M. \& Medhi, D. Determining link weight system under various objectives for OSPF networks using a Lagrangian relaxation-based approach. IEEE Transactions on Network and Service Management, 2(1):9-18, 2005.

[69] Steuer, R. E. Multiple Criteria Optimization: Theory, Computation and Application. Probability and Mathematical Statistics. John Wiley \& Sons, 1986.

[70] Talbi, E.-G., Basseur, M., Nebro, A. J. \& Alba, E. Multi-objective optimization using metaheuristics: non-standard algorithms. International Transactions in Operational Research, 19(12):283-305, 2012.

[71] Wang, J., Patek, S., Wang, H. \& Liebeherr, J. Traffic engineering with AIMD in MPLS networks. In Carle, G. \& Zitterbart, M. (editors), Proceedings of the 7th IFIP/IEEE International Workshop on Protocols for High Speed Networks (PfHSN 2002), Lecture Notes in Computer Science, volume 2334, pages 192-210, Berlin, Germany, Apr.22-24, Springer, 2002.

[72] Wang, J. Y. T. \& Ehrgott, M. Modelling stochastic route choice with bi-objective traffic assignment. In Proceedings of International Choice Modelling Conference 2011, Leeds, UK, Jul.4-6, 2011.

[73] Wang, J. Y. T. \& Ehrgott, M. Modelling route choice behavior in a tolled road network with a time surplus maximisation bi-objective user equilibrium. Procedia - Social and Behavioral Sciences, 80:266-288, 2013.

[74] Wierzbicki, A. P. \& Burakowski, W. A conceptual framework for multiple-criteria routing in QoS IP networks. International Transactions in Operational Research, 18(3):377-399, 2011.

[75] Yang, H. \& Huang, H.-J. The multiclass, multi-criteria traffic network equilibrium and systems optimum problem. Transportation Research Part B - Methodological, 38:1-15, 2004.

[76] Zhang, Q. \& Li, H. MOEA/D: A multiobjective evolutionary algorithm based on decomposition. IEEE Transactions on Evolutionary Computation, 11(6):712-731, 2007.

[77] Zitzler, E. Evolutionary multiobjective optimization. In Rozenberg, G., Bäck, T. \& Kok, J. N. (editors), Handbook of Natural Computing, pages 871-904, Springer, 2012.

[78] gt-itm. Modeling Topology of Large Internetworks, 2000. http://www.cc.gatech.edu/ projects/gtitm/ 